PROFESSOR GRIGORIS ZOIDIS (Orcid ID : 0000-0002-9442-5186)

PROFESSOR GEORGE FYTAS (Orcid ID : 0000-0003-1239-8901)

Article type : Research Article

\title{
Lipophilic conformationally constrained spiro carbocyclic 2,6- diketopiperazine-1-acetohydroxamic acid analogues as trypanocidal and leishmanicidal agents: an extended SAR study
}

Grigoris Zoidis ${ }^{1}$, Andrew Tsotinis $^{1}$, Alexandra Tsatsaroni ${ }^{1}$, Martin C. Taylor ${ }^{2}$, John M. Kelly ${ }^{2}$, Antonia Efstathiou $^{3}$, Despina Smirlis ${ }^{3}$, George Fytas ${ }^{1, *}$

${ }^{1}$ School of Health Sciences, Faculty of Pharmacy, Department of Pharmaceutical Chemistry, National and Kapodistrian University of Athens, Panepistimioupoli-Zografou, GR-15771 Athens, Greece

${ }^{2}$ Department of Pathogen Molecular Biology, London School of Hygiene and Tropical Medicine, Keppel Street, London WC1E 7HT, UK

${ }^{3}$ Laboratory of Molecular Parasitology, Department of Microbiology, Hellenic Pasteur Institute, 127 Vas. Sofias Ave, 11521 Athens, Greece

"To whom correspondence should be addressed. Phone: +302107274810 . Fax: +302107274747 . Email: gfytas@pharm.uoa.gr

\begin{abstract}
We have previously described a number of lipophilic conformationally constrained spiro carbocyclic 2,6-diketopiperazine (2,6-DKP)-1-acetohydroxamic acids as potent anti-trypanosomal agents. In this report, we extend the SAR analysis in this class of compounds with respect to in vitro growth inhibition of Trypanosoma and Leishmania parasites. Introduction of bulky hydrophobic substituents at the vicinal position of the basic nitrogen atom in the spiro carbocyclic 2,6-DKP ring system can provide analogues which are potently active against bloodstream-form $T$. brucei and exhibit significant activities towards $T$. cruzi epimastogotes and $L$. infantum promastogotes and intracellular amastigotes. In particular, compounds possessing a benzyl or 4-chlorobenzyl substituent were found to be the most active growth inhibitors, with activities in the low nanomolar and low micromolar ranges for $T$. brucei and $L$. infantum, respectively. The benzyl substituted $(S)$-enantiomer was the most potent derivative against $T$. brucei $\left(I C_{50}=6.8 \mathrm{nM}\right), T$. cruzi $\left(I C_{50}=0.21 \mu \mathrm{M}\right)$ and $L$. infantum promastigotes $\left(\mathrm{IC}_{50}=2.67 \mu \mathrm{M}\right)$ and intracellular amastigotes $\left(\mathrm{IC}_{50}=2.60 \mu \mathrm{M}\right)$. Moreover, the $(R)$-chiral benzyl substituted derivative and its racemic counterpart displayed significant activities against $L$. donovani. Importantly, the active compounds show high selectivity in comparison with two mammalian cell lines.
\end{abstract}

This article has been accepted for publication and undergone full peer review but has not been through the copyediting, typesetting, pagination and proofreading process, which may lead to differences between this version and the Version of Record. Please cite this article as doi: 10.1111/cbdd.13088

This article is protected by copyright. All rights reserved. 


\section{$1 \quad$ INTRODUCTION}

Trypanosomatid protozoan parasites of the genera Trypanosoma and Leshmania are the aetiological agents of serious diseases; human African trypanosomiasis (HAT or sleeping sickness), Chagas disease and the leishmaniases (cutaneous, mucocutaneous and visceral). These three parasitic diseases severely affect human health, representing a huge social and economic burden. As recently as the turn of the century, there were estimated to be 0.3 million infections with $T$. brucei annually, although the numbers have since fallen significantly. ${ }^{[1]}$ In Latin America, 5 - 8 million people are currently infected with $T$. cruzi, ${ }^{[2]}$ and globally there are an estimated 1 million new infections with different Leishmania species each year. ${ }^{[3]}$ Since there are no human vaccines, prevention and treatment of these devastating diseases relies on public health measures and chemotherapy. Unfortunately, the current drugs have many limitations.

Pentavalent antimony, the most widely prescribed treatment for visceral leishmaniasis, since its introduction several decades ago, has shortcomings which include serious side effects, the requirement for a prolonged course of treatment, and the emergence of drug resistance. ${ }^{[4]}$ Similarly, other visceral leishmaniasis drugs which have emerged over the past 10-15 years, also have drawbacks. With paromomycin, difficulties include administration (injectable, long treatment) and region-dependent efficacy. With miltefosine, the problems are high cost, long treatment and potential for teratogenicity. With the liposomal amphotericin B, high cost is also an issue, together with the need for hospitalization and potential teratogenicity. ${ }^{[5]}$

For HAT, the first-line treatment for second-stage infections, since its introduction in 1949, has been the arsenic-based drug melarsoprol. However, it kills $5 \%$ those treated. ${ }^{[6]}$ In addition, increased drug resistance and high therapeutic failure rates have been reported recently in several foci. ${ }^{[7]}$ Eflornithine, an alternative treatment, now given in combination with nifurtimox, is better tolerated, but difficult to administer. ${ }^{[8]}$ Despite the urgency to develop new drugs for these neglected diseases, research and development has been limited by the lack of commercial interest.

We have previously reported on a series of acetohydroxamic acid analogues (Figure 1, compounds 1a-c, 1f, 1g, 2, 6a-c, 6f, 7a, 7b) which have potent anti-trypanosome activity. ${ }^{[9]}$ These compounds were derived from conformationally constrained lipophilic spiro carbocyclic 2,6-diketopiperazine (2,6-DKP) scaffolds by introducing an acetohydroxamic acid moiety $\left(\mathrm{CH}_{2} \mathrm{CONHOH}\right)$ into their imidic nitrogen atom. The hydroxamic acid unit $(\mathrm{CONHOH})$ is indispensable for trypanocidal activity in this class of compound. ${ }^{[9]}$ Thus, we assumed that these primary hydroxamic acids act by inhibiting a vital parasite metalloenzyme via the metal ion binding action of the hydroxamate group in the catalytic site. It was also found that the potency of these acetohydroxamic acid - based trypanocidal agents was affected by: (a) the structure of the spiro carbocyclic ring and, (b) the presence of alkyl substituents on either the basic nitrogen atom (N-methylation), or at its vicinal position (Cmethylation or benzylation) in the spiro carbocyclic 2,6-DKP portion. Notably, attaching a benzyl group to the position adjacent to amine nitrogen (C-benzylation) significantly improved the potency against $T$. brucei and $T$. cruzi (Table 1 , compounds $\mathbf{1 f}, \mathbf{1} \mathbf{g}, \mathbf{2}, \mathbf{6 f}$ ) relative to the parent compounds (1a, 6a). This finding revealed that the stereoelectronic features and lipophilicity of the substituent at the above position play an important role in the potency of this class of hydroxamates. Subsequently, we

This article is protected by copyright. All rights reserved. 
have shown that the introduction of a methyl substituent into the nitrogen atom of the hydroxamic unit $(\mathrm{CONHOH})$ results in inactive compounds (Table 1, 1a, 2 vs 4,5$).{ }^{[10]}$

The present work is an extension of our ongoing efforts to enrich the structure-activity relationships based on variation of the alkyl or benzyl substituent at the position adjacent to the amine nitrogen of the 2,6-DKP ring. Thus, we incorporated at this position an isobutyl (compounds $\mathbf{1 d}, \mathbf{6 d}, \mathbf{7 d}$ ), a 2(methylthio)ethyl (compound 1e) or a 4-chlorobenzyl group (compound 3). The trypanocidal properties of the newly synthesized compounds were assessed against cultured bloodstream-form $T$. brucei. In addition, compounds $\mathbf{1 a - g , ~ 2 , ~ 3 , ~ 6 a - d , ~ 6 f , ~ 7 a , ~} \mathbf{7 b}$ and $\mathbf{7 d}$ were evaluated for their activity toward three different Leishmania sp., whilst their cytotoxicity against two established mammalian cell lines was investigated.

\section{METHODS AND MATERIALS}

\subsection{Chemistry}

Melting points were determined using a Büchi capillary apparatus and are uncorrected. ${ }^{1} \mathrm{H}$ and ${ }^{13} \mathrm{C}$ NMR spectra were obtained on Bruker MSL $400\left(400 \mathrm{MHz}{ }^{1} \mathrm{H}, 100 \mathrm{MHz}{ }^{13} \mathrm{C}\right)$, Bruker AVANCE III 600 (600 MHz ${ }^{1} \mathrm{H}, 150 \mathrm{MHz}{ }^{13} \mathrm{C}$ ), Varian $300\left(75 \mathrm{MHz}{ }^{13} \mathrm{C}\right.$ ), and Bruker AVANCE $200\left(50 \mathrm{MHz}{ }^{13} \mathrm{C}\right)$ spectrometers, using $\mathrm{CDCl}_{3}$ or DMSO- $d_{6}$ as solvent. Chemical shifts are reported in $\delta(\mathrm{ppm})$ with tetramethylsilane or solvent (DMSO- $d_{6}$ ) as internal standard. Splitting patterns are designated as $s$, singlet; $d$, doublet; $d d$, doublet of doublets; $t$, triplet; $t d$, triplet of doublets; $q$, quartet; qd, quartet of doublets; $\mathrm{m}$, multiplet; br, broad; $\mathrm{v}$ br, very broad; sym, symmetrical. The spectra were recorded at $293 \mathrm{~K}\left(20{ }^{\circ} \mathrm{C}\right)$ unless otherwise specified. Carbon multiplicities were established by DEPT experiments. 2D NMR experiments (HMQC and COSY) were performed for the elucidation of the structures of the newly synthesized compounds. Low resolution mass spectra were recorded on either an API 2000 LC-MS/MS system, using positive electrospray ionization mode or Thermo Electron Corporation DSQ mass spectrometer in chemical ionization $(\mathrm{Cl})$ in positive ion mode with methane as $\mathrm{Cl}$ reagent gas or in electron impact (EI). High resolution mass spectra (HRMS) were determined on a hybrid LTQ-Orbitrap Discovery mass spectrometer under electrospray ionization (ESI) in positive or negative ion mode. Optical rotations were measured on a Perkin Elmer 341 polarimeter at the sodium $D$ line (589). Analytical thin-layer chromatography (TLC) was conducted on precoated Merck silica gel $60 \mathrm{~F}_{254}$ plates (layer thickness $0.2 \mathrm{~mm}$ ) with the spots visualized by iodine vapors and/or UV light. Column chromatography purification was carried out on silica gel 60 (70-230 and 230-400 mesh ASTM). Elemental analyses (C, H, N) were performed by the Service Central de Microanalyse at CNRS (France) or Department of Microanalysis of NCSR "Democritos" (Greece), and were within $\pm 0.4 \%$ of the calculated values. The purities of the tested compounds were determined by analytical HPLC and elemental analysis. The obtained results correspond to $>95 \%$ purity. Analytical HPLC was performed on a Thermo Finnigan HPLC system (Thermo Finnigan, San Jose, USA) consisting of a SpectraSystem P4000 pump, a SpectraSystem 100 degasser, a SpectraSystem AS3000 autosampler, and a SpectraSystem UV2000 PDA detector, controlled by a SpectraSystem controller. ChromQuest 4.1 software was used for the management of the data. For the HPLC-DAD, a Supelco Analytical Discovery HS C18 $(250 \mathrm{~mm} \times 4.6 \mathrm{~mm}, 5.0 \mu \mathrm{m})$ column was used and the injection volume was $10 \mu \mathrm{L}$. The mobile phase consisted of $\mathrm{H}_{2} \mathrm{O}$ and $1 \%$ acetic acid (solvent A) and acetonitrile (solvent B), and solvent gradient of A/B was $95 / 5$ to $0 / 100$. The analyses were performed at r.t. with a constant flow rate of $1 \mathrm{~mL} / \mathrm{min}$ using a gradient elution of 0-50 min. The

This article is protected by copyright. All rights reserved. 
commercial reagents were purchased from Alfa Aesar, Sigma-Aldrich and Merck, and were used without further purification except for the benzyl bromoacetate. This reagent was purified by distillation in vacuo prior to use. $O$-(4-Methoxybenzyl)hydroxylamine was synthesized according to the literature reported method. ${ }^{[11]}$ Organic solvents used were in the highest purity, and when necessary, were dried by the standard methods. Yields refer to chromatographically pure materials.

The synthesis of compounds 1a-c, 1f, 1g, 2, 4, 5, 6a-c, 6f, 7a, 7b, 9, 10, 13, 17, 18, 21, 25, 31, 32, 35, $39,45,46,49,52,58,66,67,74,75,77,78,80,81,83,84$ has been previously described in our published protocols. ${ }^{[9,10,12]}$ The experimental details, physical and chemical data of compounds 14 , $15,22,23,26,28,29,36,37,40,42,43,50,51,53,55,56,59,62-64,68,69,71,72,76,79,82,85-88$ are given in the present work (Supporting information). The new compounds $\mathbf{1 d}, \mathbf{1 e}, \mathbf{3}, \mathbf{6 d}, \mathbf{7 d}, \mathbf{4 8}$, 60, 61, 65, 70, 73 (main manuscript), 11, 12, 16, 19, 20, 24, 27, 30, 33, 34, 38, 41, 44, 47, 54, 57, (Supporting Information) were synthesized as a part of this study.

\subsection{1 (S)-N-Hydroxy-2-(2-methylpropyl)-3,5-dioxospiro[piperazine-2,2'-tricyclo[3.3.1.1 ${ }^{3,7}$ ] decane]- 4-acetamide 1d}

Carboxylic acid 47 (1.33 g, $3.82 \mathrm{mmol}$ ) was treated with 1,1'-carbonyldiimidazol (743 mg, $4.58 \mathrm{mmol}$ ) in dry THF $(75 \mathrm{~mL})$ as described for the preparation of 58 from $455^{[9]}$ Then, O-benzyl hydroxylamine hydrochloride $(731 \mathrm{mg}, 4.58 \mathrm{mmol}$ ) and triethylamine $(509 \mathrm{mg}, 5.04 \mathrm{mmol}$ ) were added, and the mixture was stirred at $28^{\circ} \mathrm{C}$ for $25 \mathrm{~h}$ under argon. The reaction was worked up following the same procedure described in $\mathbf{5 8}$, and the resulting viscous oil was chromatographed on silica gel column with AcOEt- $n$-hexane 1:1, as eluent, to afford the corresponding 0 -benzyl hydroxamate $\mathbf{6 0}$ as a white foamy solid, which strongly binds the aforementioned solvents. Removal of the entrapped solvents upon drying at $62-64^{\circ} \mathrm{C}$ under vacuum $\left(10^{-3} \mathrm{mmHg}\right)$ in an Abderhalden apparatus gave 60 as a glass solid ( $1 \mathrm{~g}, 58 \%)$. This compound appears in the ${ }^{1} \mathrm{H}$ and ${ }^{13} \mathrm{C} N M R$ spectra as a mixture of $Z / E$ conformers. ${ }^{1} \mathrm{H}$ NMR $\left(600 \mathrm{MHz}, \mathrm{CDCl}_{3}, 273 \mathrm{~K}\right) \delta 0.96\left(\mathrm{t}, 6 \mathrm{H}, J=3.6,6.0 \mathrm{~Hz}, \mathrm{CH}\left(\mathrm{CH}_{3}\right) \mathrm{CH}_{3}\right), 1.05-1.30(\mathrm{br}$ s, $1 \mathrm{H}, 1-\mathrm{H}$ ), 1.39-1.60 (complex m, 3H, 4'e, 9'e-H, $\mathrm{CHHCH}\left(\mathrm{CH}_{3}\right)_{2}$ ), 1.61-1.99 (complex m, 10H, 3', 5', 6', 7', 8', 10'e-H, CHHCH(CH$)_{2}$ ), 2.05-2.20 (complex m, 2H, 1', 4'a-H), 2.42 ( t, 1H, J=12.0, $12.6 \mathrm{~Hz}, 9^{\prime} \mathrm{a}-$ H), $2.87(\mathrm{t}, 1 \mathrm{H}, J=13.2,13.8 \mathrm{~Hz}, 10 \mathrm{a}-\mathrm{H}), 3.65(\sim \mathrm{br} \mathrm{s}, 1 \mathrm{H}, 6-\mathrm{H}), 4.04-4.41$ (q, $A B, 1.2 \mathrm{H}, J_{A B}=15.0 \mathrm{~Hz}$, $\mathrm{CH}_{2} \mathrm{CONHOCH}_{2} \mathrm{Ph}$ ), 4.49-4.69 (q, $\left.\mathrm{AB}, 0.93 \mathrm{H}, \mathrm{J}_{\mathrm{AB}}=16.8 \mathrm{~Hz}, \mathrm{CH}_{2} \mathrm{CONHOCH}_{2} \mathrm{Ph}\right), 4.85,4.89$ (s+s, $2 \mathrm{H}$, $\mathrm{CONHOCH}_{2} \mathrm{Ph}$ ), 7.28-7.43 (complex m, $5 \mathrm{H}$, aromatic $\mathrm{H}$ ), $8.61\left(\mathrm{~s}, 0.38 \mathrm{H}, \mathrm{CONHOCH}_{2} \mathrm{Ph}\right.$ ), $9.03(\mathrm{~s}, 0.33 \mathrm{H}$,

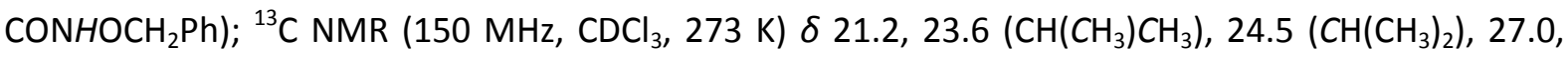
$27.3\left(5^{\prime}, 7^{\prime}-\mathrm{C}\right), 31.1\left(1^{\prime}-\mathrm{C}\right), 31.7\left(4^{\prime}-\mathrm{C}\right), 32.7$ (8'-C), 33.1 (9'-C), $33.9\left(10^{\prime}-\mathrm{C}\right), 34.9\left(3^{\prime}-\mathrm{C}\right), 37.9\left(6^{\prime}-\mathrm{C}\right), 40.3$, $40.4\left(\mathrm{CH}_{2} \mathrm{CONHOCH}_{2} \mathrm{Ph}\right), 41.7\left(\mathrm{CH}_{2} \mathrm{CH}\left(\mathrm{CH}_{3}\right)_{2}\right), 51.6(6-\mathrm{C}), 60.7\left(2,2^{\prime}-\mathrm{C}\right), 78.2,79.6\left(\mathrm{CONHOCH}_{2} \mathrm{Ph}\right)$, 128.6, 128.7, 128.9, 129.2, 129.4 (2, 3, 4, 5, 6-aromatic C), 134.2, 135.2 (1-aromatic C), 165.6, 170.8 (CONHOCH$\left.{ }_{2} \mathrm{Ph}\right), 174.9,175.9$ (3, 5-C); ESI ${ }^{+} \mathrm{MS}: \mathrm{m} / \mathrm{z} 454.5[\mathrm{M}+\mathrm{H}]^{+}$.

Compound 60 ( $856 \mathrm{mg}, 1.89 \mathrm{mmol}$ ) was subjected to catalytic hydrogenation $\left(\mathrm{H}_{2} / \mathrm{Pd}-\mathrm{C}, 103 \mathrm{mg}\right)$ in abs EtOH $(90 \mathrm{~mL})$ following the procedure previously described. ${ }^{[9]}$ The hydrogenation product was chromatographed on silica gel column using AcOEt-n-hexane 1:1, as eluent, to afford the titled compound $\mathbf{1 d}$ as a white foamy solid, which strongly binds the eluting solvents. Removal of the entrapped solvents upon drying at $55^{\circ} \mathrm{C}$ under vacuum $\left(10^{-3} \mathrm{mmHg}\right)$ in an Abderhalden apparatus gave $1 \mathrm{~d}$ as an off-white semifoamy solid (658 mg, 96\%): ${ }^{1} \mathrm{H}$ NMR (400 MHz, DMSO- $\left.d_{6}\right) \delta 0.91(\mathrm{~d}, 6 \mathrm{H}$, $\left.\mathrm{J}=6.0 \mathrm{~Hz}, \mathrm{CH}\left(\mathrm{CH}_{3}\right) \mathrm{CH}_{3}\right), 1.37$ (d, $\left.1 \mathrm{H}, \mathrm{J}=11.6 \mathrm{~Hz}, 9^{\prime} \mathrm{e}-\mathrm{H}\right), 1.41-1.51\left(\mathrm{~m}, 2 \mathrm{H}, 4\right.$ 'e-H, $\left.\mathrm{CHHCH}\left(\mathrm{CH}_{3}\right)_{2}\right), 1.53-$

This article is protected by copyright. All rights reserved. 
1.98 (complex m, 10H, 3', 5', 6', 7', 8', 10'e-H, CHHCH(CH (CH) $_{2}$ ), 2.07 (s, 1H, 1'-H), 2.14 (d, 1H, J=12.0 Hz, 4'a-H), 2.46 (d, 1H, J=11.2 Hz, 9'a-H), 2.69 (d, 1H, J=11.6 Hz, 1-H), 2.83 (d, 1H, J=10.8 Hz, 10'a-H), 3.42-3.54 ( $\mathrm{td}, 1 \mathrm{H}, J=2.6,11.1 \mathrm{~Hz}, 6-\mathrm{H}), 4.05-4.20$ (q, $\mathrm{AB}, 1.4 \mathrm{H}, \mathrm{J}_{\mathrm{AB}}=15.6 \mathrm{~Hz}, \mathrm{CH}_{2} \mathrm{CONHOH}, E$-isomer), 4.33-4.50 (q, $\mathrm{AB}, 0.4 \mathrm{H}, \mathrm{J}_{A B}=16.8 \mathrm{~Hz}, \mathrm{CH}_{2} \mathrm{CONHOH}, Z$-isomer), 8.82 (s, 0.57H, CONHOH, E-isomer), 9.24 (s, $0.14 \mathrm{H}, \mathrm{CONHOH}, Z$-isomer), $10.10(\mathrm{~s}, 0.15 \mathrm{H}, \mathrm{CONHOH}, Z$-isomer), 10.49 (s, 0.53H, CONHOH, E-

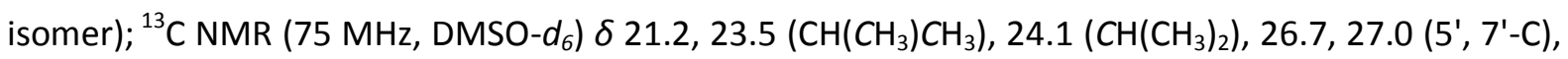
30.3, 30.5 (1'-C, Z/E-isomers), 31.3 (4'-C), 32.2 (8'-C), 32.9 (9'-C), 33.4 (10'-C), 33.9, 34.0 (3'-C, Z/Eisomers), $37.8(6 '-C), 39.6\left(\mathrm{CH}_{2} \mathrm{CONHOH}\right), 40.8,40.9\left(\mathrm{CH}_{2} \mathrm{CH}\left(\mathrm{CH}_{3}\right)_{2}, \mathrm{Z} / \mathrm{E}\right.$-isomers), 51.1 (6-C), 59.7, 59.8 (2, 2'-C, Z/E-isomers), 164.2 (CONHOH, E-isomer), 169.6 (CONHOH, Z-isomer), 174.7, 174.8, 175.0, 175.1 (3, 5-C, Z/E-isomers); $[\alpha]_{589}^{21}-29.5$ (c, 0.2, $\mathrm{CHCl}_{3}$ ); HRMS (ESI): [M-H] calc for $\mathrm{C}_{19} \mathrm{H}_{29} \mathrm{~N}_{3} \mathrm{O}_{4}$, 362.2080, found, 362.2067. The hydrochloride salt $(1 \mathbf{d} \cdot \mathbf{H C l})$ was prepared by treating an ether solution of $\mathbf{1 d}$ with ethereal $\mathrm{HCl}$ under ice cooling. The white precipitate was collected by filtration, triturated with ether and dried in vacuo. $\mathrm{Mp} 200-203^{\circ} \mathrm{C}$ (dec). Anal. Calcd for $\mathrm{C}_{19} \mathrm{H}_{30} \mathrm{ClN}_{3} \mathrm{O}_{4}$ : C, 57.06; H, 7.56; N, 10.51; Found: C, 56.72; H, 7.41; N, 10.82 .

\subsection{2}

(S)-N-Hydroxy-6-[(2-methylthio)ethyl]-3,5-dioxospiro[piperazine-2,2'-tricyclo [3.3.1.1 $1^{3,7}$ decane]-4-acetamide 1e

A mixture of benzyl ester $34(850 \mathrm{mg}, 1.86 \mathrm{mmol})$ and 10\% Pd on charcoal (1275 mg) in EtOH-AcOEt 3:2 $(70 \mathrm{~mL})$ was hydrogenated as described for the preparation of $\mathbf{4 5}$ from $\mathbf{3 1}^{[12]}$ to give a white foamy solid $\left(646 \mathrm{mg}\right.$ ). The ${ }^{1} \mathrm{H}$ NMR spectrum of the product material showed a mixture of the desired carboxylic acid $\mathbf{4 8}$ (purity ca. $91 \%$, yield $86 \%$ ) and some unchanged $\mathbf{3 4}$. The carboxylic acid $\mathbf{4 8}$ was regarded as sufficiently pure to be used in the next reaction without further purification [48: ${ }^{1} \mathrm{H}$ NMR (400 MHz, CDCl $)_{3}$ ) $1.48-1.59$ (q, 2H, J=12.4 Hz, 4'e, 9'e-H), 1.61-1.93 (complex m, 9H, $\mathrm{CHHCH}_{2} \mathrm{~S}$, $\left.3^{\prime}, 5^{\prime}, 6^{\prime}, 7^{\prime}, 8^{\prime}, 10 ' \mathrm{e}-\mathrm{H}\right), 2.11\left(\mathrm{~s}, 4 \mathrm{H}, 1^{\prime}-\mathrm{H}, \mathrm{SCH}_{3}\right), 2.18$ (d, 1H, J=12.0 Hz, 9'a-H), 2.32-2.50 (m, 2H, $\mathrm{CHHCH}_{2} \mathrm{~S}, 4$ 'a-H), 2.63-2.80 (m, $2 \mathrm{H}, \mathrm{CH}_{2} \mathrm{~S}$ ), 2.89 (d, $2 \mathrm{H}, \mathrm{J}=12.0 \mathrm{~Hz}, 10 \mathrm{a}-\mathrm{H}$ ), 3.86 (dd, $1 \mathrm{H}, J=3.4,9.0 \mathrm{~Hz}$, 6-H), 4.30-4.62 (q, $\mathrm{AB}, 2 \mathrm{H}, \mathrm{J}_{\mathrm{AB}}=17.6 \mathrm{~Hz}, \mathrm{CH}_{2} \mathrm{CO}_{2} \mathrm{H}$ ), 5.46-6.65 ( $\mathrm{v}$ br s, $2 \mathrm{H}, 1-\mathrm{H}, \mathrm{CO}_{2} \mathrm{H}$ )]. This material (646 mg) was treated with 1,1'-carbonyldiimidazol (311 mg, $1.92 \mathrm{mmol}$ ) in dry THF (32 mL) as described earlier for the preparation of $\mathbf{5 8}$ from 45. ${ }^{[9]}$ Then, 0 -benzylhydroxylamine hydrochloride (306 $\mathrm{mg}, 1.92 \mathrm{mmol}$ ) and triethylamine $(214 \mathrm{mg}, 2.11 \mathrm{mmol}$ ) were added, and the mixture was stirred at $28^{\circ} \mathrm{C}$ for $25 \mathrm{~h}$ under argon. The reaction was worked-up in exactly the same way described in 58, and the resulting viscous oil residue was chromatographed over flash silica eluting first with $\mathrm{Et}_{2} \mathrm{O}$ - $n$-hexane $1: 1$ and then AcOEt to afford the corresponding $O$-benzyl hydroxamate 61 as a colourless viscous oil, which binds the elution solvents. Removal of the entrapped solvents upon drying at $55^{\circ} \mathrm{C}$ under vacuum $\left(10^{-3} \mathrm{mmHg}\right.$ ) gave 61 as a glass solid $(542 \mathrm{mg}, 62 \%$ from 34$)$. This compound appears in the ${ }^{1} \mathrm{H}$ and ${ }^{13} \mathrm{C}$ NMR spectra as a mixture of $Z / E$ conformers. ${ }^{1} \mathrm{H}$ NMR (600 $\mathrm{MHz}, \mathrm{CDCl}_{3}, 283 \mathrm{~K}$ ) $\delta 1.38-1.47$ (br s, 1H, 1-H), 1.51 (d, 1H, J=12.4 Hz, 4'e), 1.56 (d, 1H, J=11.6 Hz, 9'eH), 1.64-1.91 (complex m, 9H, $\left.\mathrm{CHHCH}_{2} \mathrm{~S}, 3^{\prime}, 5^{\prime}, 6^{\prime}, 7^{\prime}, 8^{\prime}, 10^{\prime} \mathrm{e}-\mathrm{H}\right), 2.11$ (s, 3H, SCH$\left.)_{3}\right), 2.17$ ( br s, 2H, 1', 9'a-H), 2.34-2.50 (m, 2H, CHHCH ${ }_{2} \mathrm{~S}, 4$ 'a-H), 2.65-2.78 (m, 2H, $\left.\mathrm{CH}_{2} \mathrm{~S}\right), 2.83-2.95$ (br s, $1 \mathrm{H}, 10 \mathrm{\prime}$ a-H), 3.783.93 (br s, $1 \mathrm{H}, 6-\mathrm{H})$, 4.07-4.42 (q, $\mathrm{AB}, 1 \mathrm{H}, \mathrm{J}_{A B}=15.1 \mathrm{~Hz}, \mathrm{CH}_{2} \mathrm{CONHOCH}_{2} \mathrm{Ph}$ ), 4.54-4.73 (q, $\mathrm{AB}, 1 \mathrm{H}$, $\left.J_{A B}=16.8 \mathrm{~Hz}, \mathrm{CH}_{2} \mathrm{CONHOCH}_{2} \mathrm{Ph}\right), 4.87,4.91\left(\mathrm{~s}+\mathrm{s}, 2 \mathrm{H}, \mathrm{CONHOCH}_{2} \mathrm{Ph}\right), 7.34-7.41(\mathrm{~m}, 5 \mathrm{H}$, aromatic $\mathrm{H})$, $8.19(\mathrm{~s}, 0.44 \mathrm{H}, \mathrm{CONHOCH} 2 \mathrm{Ph}), 8.61\left(\mathrm{~s}, 0.42 \mathrm{H}, \mathrm{CONHOCH}_{2} \mathrm{Ph}\right) ;{ }^{13} \mathrm{C} \mathrm{NMR}\left(150 \mathrm{MHz}, \mathrm{CDCl}_{3}, 283 \mathrm{~K}\right) \delta 15.1$ $\left(\mathrm{SCH}_{3}\right), 26.9,27.2\left(5^{\prime}, 7^{\prime}-\mathrm{C}\right), 30.4\left(\mathrm{CH}_{2} \mathrm{~S}\right), 30.8,31.0\left(1^{\prime}-\mathrm{C}\right), 31.1\left(\mathrm{CH}_{2} \mathrm{CH}_{2} \mathrm{~S}\right), 31.4\left(9^{\prime}-\mathrm{C}\right), 32.5\left(8^{\prime}-\mathrm{C}\right), 33.1$ (4'-C), $33.8\left(10^{\prime}-\mathrm{C}\right), 35.0\left(3^{\prime}-\mathrm{C}\right), 37.9\left(6^{\prime}-\mathrm{C}\right), 40.2\left(\mathrm{CH}_{2} \mathrm{CONHOCH}_{2} \mathrm{Ph}\right), 51.7(6-\mathrm{C}), 60.9\left(2,2^{\prime}-\mathrm{C}\right), 78.1$,

This article is protected by copyright. All rights reserved. 
79.5 ( $\left.\mathrm{CONHOCH}_{2} \mathrm{Ph}\right), 128.5,128.8,129.1,129.2$ (2, 3, 4, 5, 6-aromatic C), 134.1, 135.1 (1-aromatic C), 165.5, $170.7\left(\mathrm{CONHOCH}_{2} \mathrm{Ph}\right), 174.7,174.8(3,5-\mathrm{C}) ; \mathrm{ESI}^{+} \mathrm{MS}: \mathrm{m} / \mathrm{z} 472.2[\mathrm{M}+\mathrm{H}]^{+}$.

Compound 61 ( $360 \mathrm{mg}, 0.76 \mathrm{mmol}$ ) was subjected to catalytic hydrogenation ( $10 \% \mathrm{Pd}-\mathrm{C}, 550 \mathrm{mg}$ ) in EtOH-AcOEt 3:2 $(35 \mathrm{~mL})$ following the procedure previously described. ${ }^{[9]}$ The hydrogenation material was chromatographed over flash silica eluting first with AcOEt- $n$-hexane 1:1 and then AcOEt to afford successively the unchanged compound $\mathbf{6 1}$ and title compound $1 \mathrm{e}$ as a white foamy solid, which strongly binds the elution solvents. Removal of the entrapped solvent upon drying at $62-64{ }^{\circ} \mathrm{C}$ under vacuum $\left(10^{-3} \mathrm{~mm} \mathrm{Hg}\right)$ in an Abderhalden apparatus gave 1e as an off-white solid (72 $\left.\mathrm{mg}, 25 \%\right)$ : mp 75-78 ${ }^{\circ} \mathrm{C} ;{ }^{1} \mathrm{H}$ NMR (400 MHz, DMSO- $\left.d_{6}\right) \delta 1.39$ (d, $\left.1 \mathrm{H}, J=11.7 \mathrm{~Hz}, 4^{\prime} \mathrm{e}-\mathrm{H}\right), 1.46$ (d, $1 \mathrm{H}, J=11.7 \mathrm{~Hz}$, $9^{\prime} \mathrm{e}-\mathrm{H}$ ), 1.54-1.92 (complex m, 9H, 3', 5', 6', 7', 8', 10'e- $\mathrm{H}, \mathrm{CHHCH}_{2} \mathrm{~S}$ ), 2.05 (s, 3H, $\mathrm{SCH}_{3}$ ), 2.07 (s, 1H, $\left.1^{\prime}-\mathrm{H}\right), 2.12-2.29\left(\mathrm{~m}, 2 \mathrm{H}, 9^{\prime} \mathrm{a}-\mathrm{H}, \mathrm{CHHCH}_{2} \mathrm{~S}\right), 2.45$ (d, $\left.1 \mathrm{H}, J=10.8 \mathrm{~Hz}, 4^{\prime} \mathrm{a}-\mathrm{H}\right), 2.67(\sim \mathrm{t}, 2 \mathrm{H}, J=7.3,7.8 \mathrm{~Hz}$, $\mathrm{CH}_{2} \mathrm{~S}$ ), $2.82(\sim \mathrm{d}, 2 \mathrm{H}, \mathrm{J}=11.4 \mathrm{~Hz}, 1,10$ 'a-H), 3.60-3.75 (sym quintet, $1 \mathrm{H}, 6-\mathrm{H}$ ), 4.07-4.19 (q, $\mathrm{AB}, 1.36 \mathrm{H}$, $J_{A B}=15.6 \mathrm{~Hz}, \mathrm{CH}_{2} \mathrm{CONHOH}, E$-isomer), 4.21-4.31 (m, 0.38 H, $\mathrm{CH}_{2} \mathrm{CONHOH}, E$-isomer), 4.36-4.53 (q, $\mathrm{AB}$, $0.35 \mathrm{H}, J=16.8 \mathrm{~Hz}, \mathrm{CH}_{2} \mathrm{CONHOH}, \mathrm{Z}$-isomer), 8.84 (s, $0.7 \mathrm{H}, \mathrm{CONHOH}, E$-isomer), 9.26 (s, $0.2 \mathrm{H}$, CONHOH, Z-isomer), 10.12 (s, $0.2 \mathrm{H}, \mathrm{CONHOH}, Z$-isomer), 10.52 (s, $0.7 \mathrm{H}, \mathrm{CONHOH}, E$-isomer); ${ }^{13} \mathrm{C}$ NMR (100 MHz, DMSO-d $\left.d_{6}\right) \delta 14.4\left(\mathrm{SCH}_{3}\right), 26.6,26.9\left(5^{\prime}, 7^{\prime}-\mathrm{C}\right), 29.9\left(\mathrm{CH}_{2} \mathrm{~S}\right), 30.1,30.2\left(1^{\prime}-\mathrm{C}\right), 30.8,30.9$ $\left(\mathrm{CH}_{2} \mathrm{CH}_{2} \mathrm{~S}\right), 31.1\left(9^{\prime}-\mathrm{C}\right), 32.2\left(8^{\prime}-\mathrm{C}\right), 32.8\left(4^{\prime}-\mathrm{C}\right), 33.4\left(10^{\prime}-\mathrm{C}\right), 34.0,34.1\left(3^{\prime}-\mathrm{C}\right), 37.8\left(6^{\prime}-\mathrm{C}\right), 39.5,39.9$ $\left(\mathrm{CH}_{2} \mathrm{CONHOH}\right), 51.3,51.4$ (6-C), 59.9 (2, 2'-C), 164.1 (CONHOH, E-isomer), 169.6 (CONHOH, Zisomer), 174.4, $174.6(3,5-\mathrm{C})$; $\mathrm{HRMS}\left(\mathrm{APCl}^{+}\right)$: $[\mathrm{M}+\mathrm{H}]^{+}$calcd for $\mathrm{C}_{18} \mathrm{H}_{27} \mathrm{~N}_{3} \mathrm{O}_{4} \mathrm{~S} 382,1756$, found

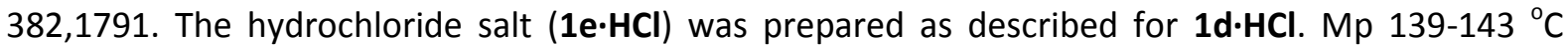
(dec); $[\alpha]_{589}^{26}-22$ (C, 0.1, DMSO). Anal Calcd for $\mathrm{C}_{18} \mathrm{H}_{28} \mathrm{CIN}_{3} \mathrm{O}_{4} \mathrm{~S}: \mathrm{C}, 51.73 ; \mathrm{H}, 6.75 ; \mathrm{N}, 10.05$. Found: $\mathrm{C}$, 52.10; $\mathrm{H}, 6.98 ; \mathrm{N}, 10.12$.

2.1.3

(RS)-6-[(4-Chlorophenyl)methyl]-N-hydroxy-2,6-dioxospiro[piperazine-2,2'-tricyclo

\section{[3.3.1.1 $1^{3,7}$ decane]-4-acetamide 3}

A mixture of 4-methoxybenzyl ester $38(1.0 \mathrm{~g}, 1.86 \mathrm{mmol})$ and TFA $(3 \mathrm{~mL})$ in $\mathrm{CH}_{2} \mathrm{Cl}_{2}(20 \mathrm{~mL})$ was stirred for $90 \mathrm{~min}$. The solution was evaporated to dryness under reduced pressure, and the residue was dissolved in dry THF (36 mL). Triethylamine (304 mg, $3 \mathrm{mmol}$ ) and 1,1'-carbonyldiimidazol (364 $\mathrm{mg}, 2.24 \mathrm{mmol}$ ) were added, and the mixture was stirred at $28^{\circ} \mathrm{C}$ for $1 \mathrm{~h}$ under argon. After this time, $\mathrm{O}$-(4-methoxybenzyl)hydroxylamine ( $344 \mathrm{mg}, 2.24 \mathrm{mmol}$ ) was added, and the mixture was stirred 24 $\mathrm{h}$ at $28^{\circ} \mathrm{C}$ and $1 \mathrm{~h}$ at $55^{\circ} \mathrm{C}$ under argon. The reaction was then worked up in the same way described for the preparation of $\mathbf{5 8}$ from $\mathbf{4 5} 5^{[9]}$. The resulting thick oil was chromatographed on silica gel column with AcOEt- $n$-hexane 1:2, as eluent, to afford the corresponding $O$-benzyl hydroxamate 65 as a white foamy solid, which strongly binds the elution solvents. Removal of the entrapped solvents as in 60 gave 65 as a glass solid (715 mg, 70\%). This compound appears in the ${ }^{1} \mathrm{H}$ and ${ }^{13} \mathrm{C}$ NMR spectra as a mixture of $E / Z$ conformers. ${ }^{1} \mathrm{H}$ NMR $\left(600 \mathrm{MHz} \mathrm{CDCl}_{3}, 273 \mathrm{~K}\right) \delta 1.39-1.50\left(\mathrm{~m}, 2 \mathrm{H}, 4^{\prime} \mathrm{e}, 9^{\prime} \mathrm{e}-\mathrm{H}\right), 1.55$ 1.88 (complex m, 10H, 1, 3', 4'a, 5', 6', '7, 8', 10'e-H), 1.99-2.17 (m, 2H, 1', 9'a-H), 2.81-3.0 (complex $\left.\mathrm{m}, 2 \mathrm{H}, 10^{\prime} \mathrm{a}-\mathrm{H}, \mathrm{CHHC}_{6} \mathrm{H}_{4} \mathrm{Cl}-4\right), 3.35\left(\sim \mathrm{d}, 1 \mathrm{H}, J=13.8 \mathrm{~Hz}, \mathrm{CHHC}_{6} \mathrm{H}_{4} \mathrm{Cl}-4\right), 3.75-3.88(\mathrm{~m}, 1 \mathrm{H}, 6-\mathrm{H}), 3.78$, 3.81 (s + s, 3H, OCH $)_{3}$, 4.10-4.40 (q, $\mathrm{AB}, 1 \mathrm{H}, \mathrm{J}_{A B}=15.3 \mathrm{~Hz}, \mathrm{CH}_{2} \mathrm{CONHOCH}_{2} \mathrm{C}_{6} \mathrm{H}_{4} \mathrm{OCH}_{3}-4$ ), 4.47-4.68 (q, $\mathrm{AB}$, $\left.1 \mathrm{H}, \mathrm{J}_{A B}=16.8 \mathrm{~Hz}, \mathrm{CH}_{2} \mathrm{CONHOCH}_{2} \mathrm{C}_{6} \mathrm{H}_{4} \mathrm{OCH}_{3}-4\right), 4.80,4.83$ ( $\left.\mathrm{s}+\mathrm{s}, 2 \mathrm{H}, \mathrm{CONHOCH}_{2} \mathrm{C}_{6} \mathrm{H}_{4} \mathrm{OCH}_{3}-4\right), 6.84-6.95$ (dd, $2 \mathrm{H}, J=8.4,20.4 \mathrm{~Hz}$, aromatic $\mathrm{H}$ ), 7.14-7.36 (complex $\mathrm{m}, 6 \mathrm{H}$, aromatic $\mathrm{H}$ ), 8.53 (s, $0.4 \mathrm{H}$, $\left.\mathrm{CONHOCH}_{2} \mathrm{C}_{6} \mathrm{H}_{4} \mathrm{OCH}_{3}-4\right), 8.96\left(\mathrm{~s}, 0.4 \mathrm{H}, \mathrm{CONHOCH} \mathrm{C}_{6} \mathrm{H}_{4} \mathrm{OCH}_{3}\right) ;{ }^{13} \mathrm{C} \mathrm{NMR}\left(150 \mathrm{MHz}, \mathrm{CDCl}_{3}, 273 \mathrm{~K}\right) \delta$ $26.9\left(5^{\prime}, 7^{\prime}-\mathrm{C}\right), 30.6\left(1^{\prime}-\mathrm{C}\right), 31.3,31.4\left(4^{\prime}-\mathrm{C}\right), 32.5\left(9^{\prime}-\mathrm{C}\right), 33.2\left(8^{\prime}-\mathrm{C}\right), 33.9\left(10^{\prime}-\mathrm{C}\right), 34.8\left(3^{\prime}-\mathrm{C}\right), 37.3\left(6^{\prime}-\right.$ C), $37.9\left(\mathrm{CH}_{2} \mathrm{C}_{6} \mathrm{H}_{4} \mathrm{Cl}-4\right)$, 40.2, $40.3\left(\mathrm{CH}_{2} \mathrm{CONHOCH}_{2} \mathrm{C}_{6} \mathrm{H}_{4} \mathrm{OCH}_{3}-4\right)$, 54.1, $54.2(6-\mathrm{C}), 55.3,55.4\left(\mathrm{OCH}_{3}\right)$,

This article is protected by copyright. All rights reserved. 
60.9 (2, 2'-C), 77.8, $79.3\left(\mathrm{CONHOCH}_{2} \mathrm{C}_{6} \mathrm{H}_{4} \mathrm{OCH}_{3}-4\right), 113.8,114.1,126.2,127.2,128.8,130.6,130.7$, 131.2, 132.8, 135.5, 159.9, 160.2 (aromatic C), 165.4, $170.6\left(\mathrm{CONHOCH}_{2} \mathrm{C}_{6} \mathrm{H}_{4} \mathrm{OCH}_{3}-4\right)$, 173.7, 174.4 (3, 5-C); $\mathrm{Cl}^{+} \mathrm{MS}: \mathrm{m} / \mathrm{z} 552.2\left([\mathrm{M}+\mathrm{H}]^{+}, 1.5\right), 426.2\left(\left[\mathrm{M}-\mathrm{CH}_{2} \mathrm{C}_{6} \mathrm{H}_{4} \mathrm{Cl}-4\right]^{+}, 8\right), 137.0(30), 121.0$ (100).

A mixture of compound 65 (451 mg, $0.82 \mathrm{mmol}$ ) and TFA (6 mL) in $\mathrm{CH}_{2} \mathrm{Cl}_{2}(23 \mathrm{~mL})$ was stirred for 10 min. The solution was then treated dropwise with $\mathrm{Et}_{3} \mathrm{SiH}(600 \mu \mathrm{L})$ via syringe, and the stirring was continued for $45 \mathrm{~min}$ at room temperature. In this time window, the colour changed from ruby to pale yellow. The reaction mixture was evaporated to dryness under reduced pressure, and the residual material was quenched with water $(15 \mathrm{~mL})$ and ether $(50 \mathrm{~mL})$ under vigorous stirring. To this stirred two phase mixture was added solid $\mathrm{Na}_{2} \mathrm{CO}_{3}$ to $\mathrm{pH}=8-9$. The organic layer was separated and the water phase was extracted with ether $(3 \times 40 \mathrm{~mL})$. The combined organics were washed with brine $(50 \mathrm{~mL})$, dried $\left(\mathrm{Na}_{2} \mathrm{SO}_{4}\right)$ and evaporated to dryness. The thick oily residue was chromatographed on silica gel column with AcOEt-n-hexane 2:1, as eluent, to afford the title compound $\mathbf{3}$ as a white foamy solid, which binds the elution solvents. Removal of the entrapped solvents as in $\mathbf{1 d}$ gave 3 as an off-white semifoamy solid (313 mg, 89\%): ${ }^{1} \mathrm{H}$ NMR (400 MHz, DMSO$\left.d_{6}\right) \delta 1.30\left(\mathrm{~d}, 2 \mathrm{H}, J=11.6 \mathrm{~Hz}, 4^{\prime} \mathrm{e}, 9^{\prime} \mathrm{e}-\mathrm{H}\right.$ ), 1.48-1.73 (complex m, 5H, 4'a, 7', 8', 10'e-H), 1.76 (s, 1H, 5'H), $1.82\left(\mathrm{~s}, 1 \mathrm{H}, 3^{\prime}-\mathrm{H}\right), 2.04\left(\mathrm{~s}, 1 \mathrm{H}, 1^{\prime}-\mathrm{H}\right), 2.18\left(\mathrm{~d}, 1 \mathrm{H}, J=12.0 \mathrm{~Hz}, 9^{\prime} \mathrm{a}-\mathrm{H}\right), 2.69-2.92$ (complex m, $3 \mathrm{H}$, $\mathrm{CHHC}_{6} \mathrm{H}_{4} \mathrm{Cl}-4,1,10^{\prime} \mathrm{a}-\mathrm{H}$ ), 3.30 (dd, $1 \mathrm{H}, \mathrm{J}=3.2,14.0 \mathrm{~Hz}, \mathrm{CHHC}_{6} \mathrm{H}_{4} \mathrm{Cl}-4$ ), 3.72 (td, $1 \mathrm{H}, \mathrm{J}=3.2,10.9 \mathrm{~Hz}, 6-\mathrm{H}$ ), 4.16 (s, $1.4 \mathrm{H}, \mathrm{CH}_{2} \mathrm{CONHOH}$ ), $E$-isomer), 4.38-4.53 (q, $\mathrm{AB}, 0.43 \mathrm{H}, J=16.8 \mathrm{~Hz}, \mathrm{CH}_{2} \mathrm{CONHOH}, Z$-isomer, 7.29-7.40 (m, $4 \mathrm{H}$, aromatic $\mathrm{H}), 8.87(\mathrm{~s}, 0.72 \mathrm{H}, \mathrm{CONHOH}, E$-isomer), $9.28(\mathrm{~s}, 0.2 \mathrm{H}, \mathrm{CONHOH}, \mathrm{Z}-$ isomer), 10.16 (s, $0.2 \mathrm{H}, \mathrm{CONHOH}, Z$-isomer), 10.55 (s, $0.7 \mathrm{H}, \mathrm{CONHOH}, E$-isomer); ${ }^{13} \mathrm{C} \mathrm{NMR} \mathrm{(50} \mathrm{MHz,}$ DMSO-d $\left.d_{6}\right) \delta 26.5\left(5^{\prime}-\mathrm{C}\right), 26.7\left(7^{\prime}-\mathrm{C}\right), 29.9\left(1^{\prime}-\mathrm{C}\right), 30.9\left(4^{\prime}-\mathrm{C}\right), 32.0\left(9^{\prime}-\mathrm{C}\right), 32.8\left(8^{\prime}-\mathrm{C}\right), 33.3\left(10^{\prime}-\mathrm{C}\right), 34.1$ (3'-C), $36.5\left(\mathrm{CH}_{2} \mathrm{C}_{6} \mathrm{H}_{4} \mathrm{Cl}-4\right), 37.7\left(6^{\prime}-\mathrm{C}\right), 39.5\left(\mathrm{CH}_{2} \mathrm{CONHOH}\right), 54.1$ (6-C), 60.0 (2, 2'-C), 127.9, 131.0 (2, 3, 5, 6-aromatic C), 130.8 (4-aromatic C), 137.8 (1-aromatic C), 164.1 (CONHOH, E-isomer), 169.5 (CONHOH, Z-isomer), 173.5., 174.5 (3,5-C); HRMS (ESI): $[\mathrm{M}+\mathrm{H}]^{+},[\mathrm{M}+\mathrm{Na}]^{+}$calcd for $\mathrm{C}_{22} \mathrm{H}_{26} \mathrm{ClN}_{3} \mathrm{O}_{4}$ 432.1690, 454.1510, found 432.1689, 454.1506. The hydrochloride salt was prepared as described for $1 \mathrm{~d} \cdot \mathrm{HCl}$. Mp $179-182{ }^{\circ} \mathrm{C}$ (dec). Anal. Calcd for $\mathrm{C}_{22} \mathrm{H}_{27} \mathrm{Cl}_{2} \mathrm{~N}_{3} \mathrm{O}_{4}: \mathrm{C}, 56.41 ; \mathrm{H}, 5.81 ; \mathrm{N}, 8.97$; Found: $\mathrm{C}$, 56.06; H, 6.12; N, 8.65 .

\subsection{4 (S)-N-Hydroxy-2-(2-methylpropyl)-3,5-dioxo-1.4-diazaspiro[5.7]tridecane-4-aceta-mide 6d}

Carboxylic acid 54 (1.13 g, $3.5 \mathrm{mmol}$ ) was treated with 1, 1'-carbonyldiimidazol (681 mg, $4.2 \mathrm{mmol}$ ) in dry THF-DMF 6:1 (70 $\mathrm{mL})$ as described for the preparation of 58 from $45{ }^{[9]}$ Then $O$ benzylhydroxylamine hydrochloride $(670 \mathrm{mg}, 4.2 \mathrm{mmol}$ ) and triethylamine $(468 \mathrm{mg}, 4.62 \mathrm{mmol})$ were added, and the mixture was stirred at $28^{\circ} \mathrm{C}$ for $25 \mathrm{~h}$ under argon. The reaction was worked up in exactly the same way described in $\mathbf{5 8}$, and the resulting viscous oil was chromatographed on silica gel column with AcOEt- $n$-hexane 2:3, as eluent, to afford the corresponding $O$-benzyl hydroxamate 70 as a white foamy solid, which strongly binds the aforementioned solvents. Removal of the entrapped solvents as in $\mathbf{6 0}$ gave 70 as a white solid (1.07 g, 71.5\%): $\mathrm{mp} 115-118^{\circ} \mathrm{C}$. This compound appears in the ${ }^{1} \mathrm{H}$ and ${ }^{13} \mathrm{C}$ NMR spectra as a mixture of $E / Z$ conformers. ${ }^{1} \mathrm{H} \mathrm{NMR}\left(400 \mathrm{MHz}, \mathrm{CDCl}_{3}\right) \delta$ 0.95 (t, 6H, J=12.6 Hz, CH(CH $\mathrm{CH}_{3}$ ), 1.10-1.27 (br s, 1H, 1-H), 1.31-2.08 (complex m, $16 \mathrm{H}, 7,8,9,10$, $\left.11,12,13-\mathrm{H}, \mathrm{CH}_{2} \mathrm{CH}\left(\mathrm{CH}_{3}\right)_{2}\right), 2.17-2.35(\mathrm{~m}, 1 \mathrm{H}, 13-\mathrm{H}), 3.62(\mathrm{~d}, 1 \mathrm{H}, \mathrm{J}=22.0 \mathrm{~Hz}, 2-\mathrm{H}), 4.12-4.40(\mathrm{br} \mathrm{s}, 1 \mathrm{H}$, $\mathrm{CH}_{2} \mathrm{CONHOCH}_{2} \mathrm{Ph}$ ), 4.45-4.75 (br s, $1 \mathrm{H}, \mathrm{CH}_{2} \mathrm{CONHOCH}_{2} \mathrm{Ph}$ ), 4.88 (s, 2H, $\mathrm{CONHOCH}_{2} \mathrm{Ph}$ ), 7.37 (s, 5H, aromatic $\mathrm{H}$ ), 8.12-8.45 (br s, 0.5H, $\left.\mathrm{CONHOCH}_{2} \mathrm{Ph}\right), 8.57-8.88$ (br s, $\left.\mathrm{CONHOCH}_{2} \mathrm{Ph}\right) ;{ }^{13} \mathrm{C} \mathrm{NMR} \mathrm{(5O} \mathrm{MHz,}$ $\left.\mathrm{CDCl}_{3}\right) \delta 20.8(9-\mathrm{C}), 21.2\left(\mathrm{CH}\left(\mathrm{CH}_{3}\right) \mathrm{CH}_{3}\right), 21.4(11-\mathrm{C}), 23.5\left(\mathrm{CH}\left(\mathrm{CH}_{3}\right) \mathrm{CH}_{3}\right), 24.5\left(10-\mathrm{C}, \mathrm{CH}\left(\mathrm{CH}_{3}\right)_{2}\right), 27.4$ (8C), 28.5 (7-C), $28.7(12-\mathrm{C}), 34.5(13-\mathrm{C}), 39.7\left(\mathrm{CH}_{2} \mathrm{CONHOCH}_{2} \mathrm{Ph}\right), 40.5\left(\mathrm{CH}_{2} \mathrm{CH}\left(\mathrm{CH}_{3}\right)_{2}\right), 51.8(2-\mathrm{C}), 60.4$

This article is protected by copyright. All rights reserved. 
(6-C), 78.4, 79.5 ( $\left.\mathrm{CONHOCH}_{2} \mathrm{Ph}\right)$, 128.7, 129.3 (2, 3, 4, 5, 6-aromatic C), 135.0 (1-aromatic C), 165.6, 170.7 ( $\left.\mathrm{CONHOCH}_{2} \mathrm{Ph}\right), 174.3,177.2$ (3, 5-C); $\mathrm{Cl}^{+} \mathrm{MS}: \mathrm{m} / \mathrm{z} 430.2\left([\mathrm{M}+\mathrm{H}]^{+}, 34\right), 429.3$ ([M] $\left.]^{+}, 13\right), 373.2$ ([M+H-CH $\left.\left.2 \mathrm{CH}\left(\mathrm{CH}_{3}\right)_{2}\right]^{+}, 18\right), 322.1\left(\left[\mathrm{M}-\mathrm{OCH}_{2} \mathrm{Ph}\right]^{+}, 18\right), 307.2$ ([M-NHOCH$\left.\left.{ }_{2} \mathrm{Ph}\right]^{+}, 22\right), 306.1$ (49), 279.2 ([M-CONHOCH$\left.\left.{ }_{2} \mathrm{Ph}\right]^{+}, 100\right), 91.0(90)$.

Compound 70 (1.19 g, $2.8 \mathrm{mmol}$ ) was subjected to catalytic hydrogenation (10\% Pd-C, $143 \mathrm{mg}$ ), in abs EtOH (126 mL) following the procedure previously described. ${ }^{[9]}$ The hydrogenation material was chromatographed on silica gel column eluting first with AcOEt- $n$-hexane 1:1 and then AcOEt to afford the title compound $\mathbf{6} \mathbf{d}$ as a white foamy solid, which strongly binds the eluting solvent. Removal of the entrapped solvent as in 1 e gave $\mathbf{6 d}$ as an off-white solid (850 mg, 90.5\%): $\mathrm{mp} 129$ $132^{\circ} \mathrm{C}$; ${ }^{1} \mathrm{H}$ NMR $\left(400 \mathrm{MHz}\right.$, DMSO- $\left.d_{6}\right) \delta 0.84\left(\mathrm{~d}, 3 \mathrm{H}, J=6.4 \mathrm{~Hz}, \mathrm{CH}\left(\mathrm{CH}_{3}\right) \mathrm{CH}_{3}\right), 0.88(\mathrm{~d}, 3 \mathrm{H}, J=6.4 \mathrm{~Hz}$, $\mathrm{CH}\left(\mathrm{CH}_{3}\right) \mathrm{CH}_{3}$ ), 1.30-1.96 (complex m, 16H, 7, 8, 9, 10, 11, 12, 13- $\left.\mathrm{H}, \mathrm{CH}_{2} \mathrm{CH}\left(\mathrm{CH}_{3}\right)_{2}\right), 1.97-2.10$ (q, $1 \mathrm{H}$, $J=9.2 \mathrm{~Hz}, 13-\mathrm{H}), 2.42-2.48(\mathrm{~m}, 1 \mathrm{H}, 1-\mathrm{H}), 3.36-3.53(\mathrm{~m}, 1 \mathrm{H}, 2-\mathrm{H}), 4.0-4.13\left(\mathrm{q}, \mathrm{AB}, 1.5 \mathrm{H}, J_{A B}=15.6 \mathrm{~Hz}\right.$, $\mathrm{CH}_{2} \mathrm{CONHOH}, E$-isomer), 4.30-4.42 (q, $\mathrm{AB}, 0.4 \mathrm{H}, J_{A B}=16.8 \mathrm{~Hz}, \mathrm{CH}_{2} \mathrm{CONHOH}, Z$-isomer), 8.79 (s, $0.8 \mathrm{H}$, CONHOH, E-isomer), 9.21 (s, 0.2H, CONHOH, Z-isomer), 10.08 (s, 0.2H, CONHOH, Z-isomer), 10.43 (s, 0.7H, CONHOH, E-isomer); ${ }^{13} \mathrm{C} N M R\left(75 \mathrm{MHz}, \mathrm{DMSO}-d_{6}\right) \delta 20.4$ (9-C), 21.0 (11-C), 21.123 .5 $\left(\mathrm{CH}\left(\mathrm{CH}_{3}\right) \mathrm{CH}_{3}\right), 24.0\left(\mathrm{CH}\left(\mathrm{CH}_{3}\right)_{2}\right), 24.2(10-\mathrm{C}), 27.0(8-\mathrm{C}), 27.7,27.8$ (7-C), 28.3 (12-C), $33.4(13-\mathrm{C}), 39.2$, $39.3\left(\mathrm{CH}_{2} \mathrm{CONHOH}\right), 39.6\left(\mathrm{CH}_{2} \mathrm{CH}\left(\mathrm{CH}_{3}\right)_{2}\right), 51.1(2-\mathrm{C}), 59.5(6-\mathrm{C}), 164.0(\mathrm{CONHOH}, E$-isomer), 169.5 (CONHOH, Z-isomer), 174.1, $176.9(3,5-\mathrm{C}) ;[\alpha]_{589}^{23}-11$ (c, 0.2, $\mathrm{CHCl}_{3}$ ); HRMS (ESI): [M-H] calcd for $\mathrm{C}_{17} \mathrm{H}_{29} \mathrm{~N}_{3} \mathrm{O}_{4}, 338.2080$, found, 338.2069. The hydrochloride salt $(\mathbf{6 d} \cdot \mathbf{H C l})$ was prepared as described for $1 \mathrm{~d} \cdot \mathrm{HCl}$. Mp 212-214 ${ }^{\circ} \mathrm{C}$ (dec). Anal. Calcd for $\mathrm{C}_{17} \mathrm{H}_{30} \mathrm{ClN}_{3} \mathrm{O}_{4}: \mathrm{C}, 54.32 ; \mathrm{H}, 8.05 ; \mathrm{N}, 11.18$; Found: $\mathrm{C}$, 53.98; H, 7.88; N, 10.89 .

\subsection{5 (S)-N-Hydroxy-2-(2-methylpropyl)-3,5-dioxospiro-1,4-diazaspiro[5.6]dodecane-4-acetamide $7 d$}

Carboxylic acid 57 (1.52 g, $4.9 \mathrm{mmol}$ ) was treated with 1,1'-carbonyldiimidazol (957 mg, $5.9 \mathrm{mmol}$ ) in dry THF-DMF 6:1 $(100 \mathrm{~mL})$ as described for the preparation of 58 from 45. ${ }^{[9]}$ Then, $O$ benzylhydroxylamine hydrochloride $(942 \mathrm{mg}, 5.9 \mathrm{mmol})$ and triethylamine $(657 \mathrm{mg}, 6.5 \mathrm{mmol})$ were added, and the mixture was stirred at $28^{\circ} \mathrm{C}$ for $25 \mathrm{~h}$ under argon. The reaction was worked up in exactly the same way described in $\mathbf{5 8}$, and the resulting thick oil was chromatographed on silica gel column with AcOEt- $n$-hexane 2:3, as eluent, to afford the corresponding $O$-benzyl hydroxamate $\mathbf{7 3}$ as a white foamy solid, which strongly binds the aforementioned solvents. Removal of the entrapped solvents as in 60 gave 73 as a white solid $(1.54 \mathrm{~g}, 76 \%): 129-131^{\circ} \mathrm{C}$. This compound appears in the ${ }^{1} \mathrm{H}$ and ${ }^{13} \mathrm{C} \mathrm{NMR}$ spectra as a mixture of $E / Z$ conformers. ${ }^{1} \mathrm{H} \mathrm{NMR}\left(400 \mathrm{MHz}, \mathrm{CDCl}_{3}\right) \delta 0.93(\mathrm{~d}, 3 \mathrm{H}, \mathrm{J}=6.0$ $\left.\mathrm{Hz}, \mathrm{CH}\left(\mathrm{CH}_{3}\right) \mathrm{CH}_{3}\right), 0.96$ (d, 3H, J=6.3 Hz, CH( $\left.\mathrm{CH}_{3}\right) \mathrm{CH}_{3}$ ), 1.13-1.37 (br s, $1 \mathrm{H}, 1-\mathrm{H}$ ), 1.38-2.03 (complex m, $\left.14 \mathrm{H}, 7,8,9,10,11,12-\mathrm{H}, \mathrm{CH}_{2} \mathrm{CH}\left(\mathrm{CH}_{3}\right)_{2}\right), 2.27$ ( $\left.\mathrm{t}, 1 \mathrm{H}, \mathrm{J}=9.9,12.0 \mathrm{~Hz}, 12-\mathrm{H}\right), 3.60$ (d, $1 \mathrm{H}, \mathrm{J}=8.0 \mathrm{~Hz}, 2-\mathrm{H}$ ), 4.09-4.39 (br s, $\left.1 \mathrm{H}, \mathrm{CH}_{2} \mathrm{CONHOCH}_{2} \mathrm{Ph}\right), 4.45-4.69$ (br s, $\left.1 \mathrm{H}, \mathrm{CH}_{2} \mathrm{CONHOCH}_{2} \mathrm{Ph}\right), 4.87$ (s, $2 \mathrm{H}$, $\mathrm{CONHOCH}_{2} \mathrm{Ph}$ ), 7.37 (s, 5H, aromatic H), 8.24-8.44 (br s, 0.34H, $\left.\mathrm{CONHOCH}_{2} \mathrm{Ph}\right), 8.75-8.93(\mathrm{~s}, 0.37 \mathrm{H}$,

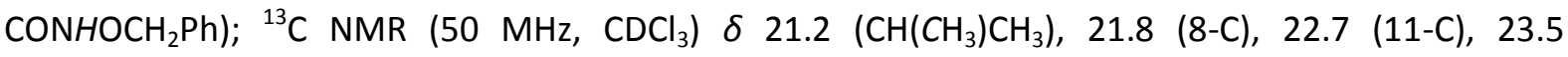
$\left(\mathrm{CH}\left(\mathrm{CH}_{3}\right) \mathrm{CH}_{3}\right), 24.5\left(\mathrm{CH}\left(\mathrm{CH}_{3}\right)_{2}\right), 29.4,29.6(9,10-\mathrm{C}), 33.6(7-\mathrm{C}), 38.8(12-\mathrm{C}), 39.8\left(\mathrm{CH}_{2} \mathrm{CONHOCH}_{2} \mathrm{Ph}\right)$, $40.3\left(\mathrm{CH}_{2} \mathrm{CH}\left(\mathrm{CH}_{3}\right)_{2}\right), 51.8(2-\mathrm{C}), 61.2(6-\mathrm{C}), 78.5,79.6\left(\mathrm{CONHOCH}_{2} \mathrm{Ph}\right), 128.8,129.4(2,3,4,5,6-$ aromatic C), 134.9 (1-aromatic C), 165.7, $170.8\left(\mathrm{CONHOCH}_{2} \mathrm{Ph}\right), 174.3,177.6(3,5-\mathrm{C}) ; \mathrm{Cl}^{+} \mathrm{MS}: \mathrm{m} / \mathrm{z}$ $416.2\left([\mathrm{M}+\mathrm{H}]^{+}, 5\right), 359.1\left(\left[\mathrm{M}+\mathrm{H}-\mathrm{CH}_{2} \mathrm{CH}\left(\mathrm{CH}_{3}\right)_{2}\right]^{+}, 5\right), 265.1$ ([M-CONHOCH$\left.\left.{ }_{2} \mathrm{Ph}\right]^{+}, 18\right), 264.1$ (59), 91.0 (100).

This article is protected by copyright. All rights reserved. 
Compound 73 (1.62 g, $3.9 \mathrm{mmol}$ ) was subjected to catalytic hydrogenation (10\% Pd-C, $194 \mathrm{mg}$ ), in abs EtOH (175 mL) following the procedure previously described. ${ }^{[9]}$ The hydrogenation material was chromatographed on silica gel column with AcOEt-n-hexane 3:1, as eluent, to afford the title compound $\mathbf{7 d}$ as a white foamy solid, which strongly binds the aforementioned solvents. Removal of the entrapped solvent as in $1 \mathbf{e}$ gave $\mathbf{7 d}$ as an off-white crystalline solid $(1.18 \mathrm{~g}, 93 \%)$ : $\mathrm{mp} 152-155^{\circ} \mathrm{C}$; ${ }^{1} \mathrm{H}$ NMR $\left(400 \mathrm{MHz}\right.$, DMSO- $\left.d_{6}\right) \delta 0.88\left(\mathrm{~d}, 3 \mathrm{H}, J=6.4 \mathrm{~Hz}, \mathrm{CH}\left(\mathrm{CH}_{3}\right) \mathrm{CH}_{3}\right), 0.92(\mathrm{~d}, 3 \mathrm{H}, J=6.8 \mathrm{~Hz}$, $\mathrm{CH}\left(\mathrm{CH}_{3}\right) \mathrm{CH}_{3}$ ), 1.33-1.80 (complex $\mathrm{m}, 12 \mathrm{H}, 7,8,9,10,11,12-\mathrm{H}, \mathrm{CH}_{2} \mathrm{CH}\left(\mathrm{CH}_{3}\right)_{2}$ ), 1.83-2.0 (m, 2H, $\left.\mathrm{CH}\left(\mathrm{CH}_{3}\right)_{2}, 7-\mathrm{H}\right), 2.02-2.16(\mathrm{~m}, 1 \mathrm{H}, 12-\mathrm{H}), 2.59(\mathrm{~d}, 1 \mathrm{H}, \mathrm{J}=12.4 \mathrm{~Hz}, 1-\mathrm{H}), 3.41-3.56(\mathrm{~m}, 1 \mathrm{H}, 2-\mathrm{H}), 4.06-$ 4.19 (q, $A B, 1.5 \mathrm{H}, J_{A B}=15.6 \mathrm{~Hz}, \mathrm{CH}_{2} \mathrm{CONHOH}, E$-isomer), 4.35-4.51 (q, $\mathrm{AB}, 0.4 \mathrm{H}, J_{A B}=16.8 \mathrm{~Hz}$, $\mathrm{CH}_{2} \mathrm{CONHOH}, \mathrm{Z}$-isomer), 8.84 (s, 0.7H, CONHOH, E-isomer), 9.25 (s, 0.2H, CONHOH, Z-isomer), 10.13 (s, 0.2H, CONHOH, Z-isomer), 10.48(s, 0.7H, CONHOH, E-isomer); $\left.{ }^{13} \mathrm{C} \mathrm{NMR} \mathrm{(75} \mathrm{MHz,} \mathrm{DMSO-d} d_{6}\right) \delta 21.1$ $\left(\mathrm{CH}\left(\mathrm{CH}_{3}\right) \mathrm{CH}_{3}\right), 21.3(8-\mathrm{C}), 22.3(11-\mathrm{C}), 23.5\left(\mathrm{CH}\left(\mathrm{CH}_{3}\right) \mathrm{CH}_{3}\right), 24.0\left(\mathrm{CH}\left(\mathrm{CH}_{3}\right)_{2}\right), 29.1,29.3(9,10-\mathrm{C}), 33.1$ (7C), $37.7(12-\mathrm{C}), 39.4\left(\mathrm{CH}_{2} \mathrm{CONHOH}\right), 39.5\left(\mathrm{CH}_{2} \mathrm{CH}\left(\mathrm{CH}_{3}\right)_{2}\right), 51.1(2-\mathrm{C}), 60.2,60.3(6-\mathrm{C}), 164.1$ (CONHOH, E-isomer), 169.5 (CONHOH, Z-isomer), 174.1, 177.4 (3, 5-C); $[\alpha]_{589}^{23}-21$ (c, 0.2, $\mathrm{CHCl}_{3}$ ); HRMS (ESI): $[\mathrm{M}-\mathrm{H}]^{-}$calcd for $\mathrm{C}_{16} \mathrm{H}_{27} \mathrm{~N}_{3} \mathrm{O}_{4}, 324.1923$, found, 324.1911. The hydrochloride salt $(\mathbf{7 d} \cdot \mathbf{H C l})$ was prepared as described for $1 \mathbf{d} \cdot \mathbf{H C l}$. $\mathrm{Mp} 211-213^{\circ} \mathrm{C}$ (dec). Anal. Calcd for $\mathrm{C}_{16} \mathrm{H}_{28} \mathrm{ClN}_{3} \mathrm{O}_{4}: \mathrm{C}, 53.10 ; \mathrm{H}$, 7.80; N, 11.61; Found: C, 53.39; H, 7.65; N, 11.32 .

\subsection{Biological evaluation}

\subsubsection{Trypanocidal assays}

Bloodstream form T. brucei (strain 221) were cultured in modified Iscove's medium, as outlined previously. ${ }^{[13]}$ Assays were performed in 96-well microtitre plates and the compound concentrations which inhibited growth by $50 \%\left(\mathrm{IC}_{50}\right)$ and $90 \%\left(\mathrm{IC}_{90}\right)$ were determined. Parasites were first diluted to $2.5 \times 10^{4} \mathrm{~mL}^{-1}$, compounds were added at range of concentrations, and the plates incubated at $37^{\circ} \mathrm{C}$. Resazurin was added after $48 \mathrm{~h}(12.5 \mu \mathrm{g} / \mathrm{ml})$, the plates incubated for a further $16 \mathrm{~h}$, and then read in a Spectramax plate reader (excitation $555 \mathrm{~nm} /$ emission $585 \mathrm{~nm}$ ). Results were analysed using GraphPad Prism. Each drug concentration was tested in triplicate.

T. cruzi epimastogotes (strain CL Brener) were cultured as described. ${ }^{[14]}$ Trypanocidal activity was determined in microtitre plates as outlined above, with the following modifications. Experiments were initiated by seeding the parasites at $2.5 \times 10^{5} \mathrm{~mL}^{-1}$, and after the addition of test compounds, cultured at $28^{\circ} \mathrm{C}$ for 4 days. Resazurin was added, the plates were incubated for a further 2 days, and then assessed as above.

\subsubsection{Leishmanicidal assays and cytotoxicity assays on murine macrophages J774.1. cells}

L. donovani (strain LG13, MHOM/ET/0000/HUSSEN), L. infantum (MHOM/GR/2002/GH12) and L. amazonensis (MPRO/BR/72/M1845) promastigotes were cultured at $26{ }^{\circ} \mathrm{C}$ in RPMI 1640 (RPMI) medium supplemented with $10 \%$ heat-inactivated fetal bovine serum (fully supplmented RPMI), 10 $\mathrm{mM}$ HEPES and antibiotics (penicillin/ streptomycin) as previously described. ${ }^{[15]}$ The murine macrophage J774.1 cell line (American Type Culture Collection, Manassas, VA) was cultured as well as described above, in fully supplemented RPMI, and incubated at $37{ }^{\circ} \mathrm{C}$ in $5 \% \mathrm{CO}_{2}$. The inhibitory activity and the cytotoxicity of the compounds were determined with the use of an MTT-based assay, the Alamar blue, as previously described. ${ }^{[16]}$ More specifically, $2.5 \times 10^{6}$ parasites $/ \mathrm{ml}$ and $2 \times 10^{5}$ macrophages $/ \mathrm{ml}$ were seeded into 96 -well plates (in total volume $200 \mu \mathrm{l}$ ) and they were incubated for 72 hours with different concentrations of the compounds at $26{ }^{\circ} \mathrm{C}$ and $37{ }^{\circ} \mathrm{C}$ (in $5 \% \mathrm{CO}_{2}$ )

This article is protected by copyright. All rights reserved. 
respectively. $20 \mu \mathrm{l}$ of alamar blue were added upon the 72 hour incubation and 24 hours later the colorimetric readings were performed (wavelength: $550 \mathrm{~nm}$, reference wavelength: $620 \mathrm{~nm}$ ). The $50 \%$ maximal inhibitory concentration $\left(\mathrm{IC}_{50}\right)$ was calculated using a nonlinear regression curve fit. ${ }^{[17]}$ For evaluating the inhibitory activity of compounds against intracellular amastigotes, J774.1 macrophages were seeded into 96 -well flat bottom plates at a density of $2 \times 10^{5}$ cells $/ \mathrm{mL}$ and a total volume of $200 \mu \mathrm{l}$, and were left to adhere overnight at $37{ }^{\circ} \mathrm{C}$ in $5 \% \mathrm{CO}_{2}$. Then, the macrophages were infected with stationary phase $4 \times 10^{6}$ promastigotes $/ \mathrm{ml}$, at a ratio of 10 parasites per 1 macrophage, and incubated for a further $24 \mathrm{~h}$ at $37{ }^{\circ} \mathrm{C}$ in $5 \% \mathrm{CO}_{2}$ as previously described. ${ }^{[16]}$ Upon the incubation, the medium was removed, wells were thoroughly washed with PBS to remove free parasites and $200 \mu \mathrm{l}$ of fresh medium containing the different concentrations of the compounds was added. The plate was incubated for further 72 hours at $37{ }^{\circ} \mathrm{C}$ in $5 \% \mathrm{CO}_{2}$ and $20 \mu \mathrm{l}$ of alamar blue was added as described above. All experiments were performed at least three independent times in triplicate.

\subsubsection{Cytotoxicity assays on rat skeletal myoblast $\mathbf{L} 6$ cells}

Cytotoxicity against L6 cells was assessed using microtitre plates. Briefly, cells were seeded in triplicate at $1 \times 10^{4} \mathrm{~mL}^{-1}$ in growth medium containing different compound concentrations. The plates were incubated for 6 days at $37^{\circ} \mathrm{C}$ and resazurin then added to each well. After a further $8 \mathrm{~h}$ incubation, the fluorescence was determined using a Spectramax plate reader.

\section{RESULTS AND DISCUSSION}

\subsection{Chemistry}

The synthetic routes for the preparation of the target compounds (1a-g, 2-5, 6a-d, 6f, 7a, 7b and 7d) is illustrated in Scheme 1. 2,6-Diketopiperazine-1-acetic acids 45-57 and 83-85 were the pivotal intermediates in preparing the acetohydroxamic acid analogues 1a-g, 2, 4, 5, 6a-d, 6f, 7a, 7b and 7d. Thus, coupling of these carboxylic acids with $\mathrm{O}$-benzylhydroxylamine or $\mathrm{O}$-benzyl- $\mathrm{N}$ methylhydroxylamine in the presence of $1,1^{\prime}$-carbonyldiimidazol (CDI) in THF or THF-DMF mixtures afforded the corresponding $O$-benzyl hydroxamates $58-64,66-73$ and $86-88$ in yields $58-95 \%$. Subsequent benzyl deprotection by hydrogenation over $10 \% \mathrm{Pd}-\mathrm{C}$ gave the respective hydroxamic acids $\mathbf{1 a - g}, \mathbf{2}, \mathbf{4}, \mathbf{5}, \mathbf{6 a - d}, \mathbf{6 f}, \mathbf{7 a}, \mathbf{7 b}$ and $\mathbf{7 d}$. It is of note that this reductive deprotection provided $\mathbf{1 a -}$ d, 1f, 1g, 2, 4, 5, 6a-d, 6f, 7a, 7b and 7d in high yields (80-96\%), whilst it was low yielding (<10\%) for 1e. This is presumably due to catalyst poisoning by the methionine sulfide group. A better yield of $\mathbf{1 e}$ (25\%) was achieved when the catalyst amount was 1.5 -fold the weight of its corresponding $O$-benzyl hydroxamate precursor 61.

The preparation of the chloro substituted hydroxamic acid $\mathbf{3}$ needed an alternative synthetic process as shown in Scheme 1. 4-Methoxybenzyl ester $\mathbf{3 8}$ was treated with trifluoroacetic acid (TFA) to give the respective carboxylic acid (not shown), which was coupled with $O-(4-$ methoxybenzyl)hydroxylamine in the presence of $\mathrm{CDI}$ to form the $O$-(4-methoxybenzyl) hydroxamate 65 (70\% from 38). Removal of the 4-methoxybenzyl protecting group of 65 was effected by treatment with trifluoroacetic acid in the presence of triethylsilane to give the desired compound 3 in $\mathbf{8 9 \%}$ yield. The requisite 4-methoxybenzyl ester $\mathbf{3 8}$ was synthesized from $\mathbf{2 4}$ as described for the preparation of the benzyl esters 31-37 and 39-44, except that 4-methoxybenzyl bromoacetate was used in place of benzyl bromoacetate. It is noteworthy that the 4-methoxybenzyl bromoacetate ${ }^{[18,19]}$ used was prepared by employing a new facile and efficient experimental protocol involving the

This article is protected by copyright. All rights reserved. 
treatment of 4-methoxybenzyl alcohol with bromoacetyl chloride in the presence of $\mathrm{NaHCO}_{3}$ in dry $\mathrm{CH}_{2} \mathrm{Cl}_{2}$ (Supporting Information).

The ${ }^{1} \mathrm{H}$ and ${ }^{13} \mathrm{C}$ NMR spectra for all acetohydroxamic acid analogues (compounds 1a-g, 2-5, 6a-d, 6f, $\mathbf{7 a}, \mathbf{7 b}$ and $\mathbf{7 d}$ ) are consistent with a $Z / E$ conformational behavior of these molecules in solution. The NMR spectral data of compounds $\mathbf{1 a - c}, \mathbf{1 f}, \mathbf{1 g}, \mathbf{2}, \mathbf{6 a - c}, \mathbf{6 f}, \mathbf{7 a}$ and $\mathbf{7 b}$ have already been reported. ${ }^{[9]}$ However, the assignment of the $Z$ and $E$ conformers given in this report was erroneous. Our next, more detailed conformational study, ${ }^{[10]}$ has reversed these assignments, i.e. the original data referring to the $E$ isomer correspond to the $Z$ isomer structure and vice versa.

\subsection{Biological evaluation}

Compounds 1a-g, 2-5, 6a-d, 6f, 7a, 7b and 7d were tested against bloodstream form T. brucei in vitro. Analogues $\mathbf{1} \mathbf{a}, \mathbf{1} \mathbf{b}$ and $\mathbf{1} \mathbf{f}$ were also tested against $T$. cruzi epimastigotes. The results are shown in Table 1 and expressed as $\mathrm{IC}_{50}$ and $\mathrm{IC}_{90}$ values. The in vitro anti-leishmanial activity of compounds 1a-g, 2, 3, 6a-d, 6f, 7a, 7b and $\mathbf{7 d}$ was evaluated against promastigote and intracellular amastigote forms of $L$. infantum GH12, L. donovani LG13 and L. amazonensis LV78. The IC ${ }_{50}$ values are given in Table 2. The cytotoxicities of the hydroxamic acid derivatives against mammalian cells were determined using the rat skeletal myoblast L6 and murine macrophage J 174.1 cell lines.

Inspection of Table 1 shows that compounds $\mathbf{1 a - g}, \mathbf{2}, \mathbf{3}, \mathbf{6 a - d}, \mathbf{6 f}, \mathbf{7 a}, \mathbf{7 b}$ and $\mathbf{7 d}$ exhibited potent activity against $T$. brucei as either free bases or hydrochloride salts $\left(\mathrm{IC}_{50}=6.6-1870 \mathrm{nM}\right.$ and $9.2-1150$ $\mathrm{nM}$ respectively). In particular, compounds $\mathbf{1 f}, \mathbf{1} \mathbf{g}, \mathbf{2}, \mathbf{3}, \mathbf{6} \mathbf{d}$ and $\mathbf{6 f}$ were the most potent against African trypanosomes, with $\mathrm{IC}_{50}$ values in the low nanomolar range (6.8-72 $\left.\mathrm{nM}\right)$, while hydroxamates 1a, $\mathbf{1 b}$ and $\mathbf{1 f}$ were found to be significantly active against $T$. cruzi epimastigotes. Structure-activity relationship studies show that the substitution pattern of the spiro carbocyclic 2,6-DKP scaffold is an essential factor in the trypanocidal potency of these acetohydroxamic acid-based analogues.

Methylation on the basic nitrogen of the 2,6-DKP ring of the adamantane parent compound $\mathbf{1 a}$ resulted in 2.1-fold less activity against $T$. brucei (compare 7b vs 7a). However, analogous methylation on the cyclooctane and cycloheptane parent molecules $6 a$ and $7 a$ provided a 1.9 and 6.6-fold increase in the potency of their $N$-methyl analogues $\mathbf{6 b}$ and $\mathbf{7 b}$, respectively (Table 1 ). These results indicate that $\mathrm{N}$-methyl substitution on the 2,6-DKP ring has a favorable effect on the trypanocidal activity only in the cases of the cyclooctane or cycloheptane-containing acetohydroxamic acid analogues in this series of compounds.

\section{Table 1.}

We next investigated the effect of alkyl or arylmethyl substitution at the vicinal position of the basic nitrogen atom within the spiro carbocyclic 2,6-DKP framework, using methyl, isobutyl, 2(methylthio)ethyl, benzyl and 4-chlorobenzyl substituents. As shown in Table 1, introduction of a methyl, isobutyl or 2-(methylthio)ethyl substituent to the adamantane parent 1a, leading to the $(S)$ enantiomer of the corresponding $\mathrm{C}$-alkylated analogues $\mathbf{1 c}, \mathbf{1} \mathbf{d}$ and $\mathbf{1 e}$, reduced potency against $T$. brucei. Compounds $\mathbf{1 c}, \mathbf{1} \mathbf{d}$ and $\mathbf{1 e}$, in the free base form, were 1.5, 3.1 and 1.9-fold less potent than 1a. The lowest activity relative to $\mathbf{1 a}$ resulted from the introduction of an isobutyl group (1d). However, similar C-methylation or isobutylation of the cyclooctane parent molecule 6a appeared to

This article is protected by copyright. All rights reserved. 
have a beneficial effect on the trypanocidal potency; the resulting (S)-methyl and (S)-isobutyl substituted analogues $\mathbf{6 c}$ and $\mathbf{6 d}$ had 2.4 and 4.2 -fold better activities than $\mathbf{6 a}$, respectively. It is interesting that a significantly improved activity (19-fold) was observed with the cycloheptanecontaining $(S)$-isobutyl substituted congener $\mathbf{7 d}$ relative to the parent compound $\mathbf{7 a}$. Therefore, the presence of a methyl or isobutyl group at the vicinal position of the amine nitrogen atom in the 2,6DKP ring seems to favor trypanocidal potency toward $T$. brucei in the context of the spiro cyclooctane or cycloheptane 2,6-diketopiperazine-1-acetohydroxamic acids.

Attachment of a bulky hydrophobic substituent, such as a benzyl or 4-chlorobenzyl group, to the methylene carbon in the 2,6-DKP ring of the parent molecules $1 \mathbf{a}$ and $\mathbf{6 a}$ led to the most potent analogues (1f, 1g, 2, $\mathbf{3}$ and $\mathbf{6 f}$ ) in this series against bloodstream-form $T$. brucei, with activity in low nanomolar range $\left(\mathrm{IC}_{50}=6.8-32 \mathrm{nM}\right.$, Table 1$)$. Of the adamantane-based compounds possessing a benzyl substituent, the $S$-enantiomer $\left(\mathbf{1} \mathbf{f}, \mathrm{IC}_{50}=6.8 \mathrm{nM}\right)$ was more potent than the $R$-enantiomer counterpart $\left(1 \mathrm{~g}, I C_{50}=9.1 \mathrm{nM}\right)$, or racemate $\left(2, I C_{50}=17 \mathrm{nM}\right)$. Their activities in the free base form were found to be 5.3-13 times higher than the unsubstituted parent 1a and 8-42 times higher than their C-menthyl and C-isobutyl substituted congeners $\mathbf{1 c}$ and $\mathbf{1 d}$. Incorporation of a 4-chlorobenzyl substituent at the same position of the parent $1 \mathbf{a}$, leading to the racemate p-chlorobenzyl substituted derivative 3, caused an increase in activity (2.8-fold), albeit to a lesser extent than the respective benzylic substitution (racemate 2 vs 3 ). A similar potency profile was observed for the cyclooctane C-benzyl substituted analogue $\mathbf{6 f}$ ( $S$-enantiomer). This compound was significantly more potent (10-fold) than the parent $6 \mathbf{6}$, and had 4.3 and 2.5 more trypanocidal activity than the $(S)$ enantiomers of the C-methyl and C-isobutyl counterparts $\mathbf{6 c}$ and $\mathbf{6 d}$, respectively (Table 1 ). The higher increase in potency of the C-benzylated analogues $\mathbf{1 f}, \mathbf{1 g}, \mathbf{2}, \mathbf{3}$ and $\mathbf{6} \mathbf{f}$ is ascribed to the strongly favorable stereoelectronic and lipophilic effects exerted by the benzylic substituent in the binding site.

The adamantane-containing free bases $\mathbf{1 a}, \mathbf{1 b}$ and $\mathbf{1} \mathbf{f}$ were significantly active against cultured $T$. cruzi epimastigotes, with micromolar to submicromolar $\mathrm{IC}_{50}$ values (Table 1 ). It is noteworthy, that $\mathrm{N}$ methylation in analogue $\mathbf{1 b}$ leads to an opposite effect to that in $T$. brucei. Similarly, $\mathbf{C}$-benzylation on 1a, giving compound $\mathbf{1 f}$, led to a much more potency against $T$. cruzi (26-fold). ${ }^{\left[{ }^{[9]}\right.}$

The results of the in vitro assay for the anti-leishmanial activity of compounds $\mathbf{1 a - g , 2 , 3 , 6 a - d , ~ 6 f , ~ 7 a , ~}$ $\mathbf{7 b}$ and $\mathbf{7 d}$ are presented in Table 2 . These compounds were first screened against the promastigote form of each of the three Leishmania sp. at $10 \mu \mathrm{M}$. IC $\mathrm{C}_{50}$ values were further determined for those displaying $100 \%$ parasite growth inhibition at this concentration.

The unsubstituted parent compounds $\mathbf{1 a}, \mathbf{6 a}, \mathbf{7 a}$ and their substituted derivatives $\mathbf{1 b}, \mathbf{1 c}, \mathbf{1 e}, \mathbf{6 b}, \mathbf{6 c}$, 6f and $\mathbf{7 b}$ had a marginal activity toward all the Leishmania sp. tested. In contrast, compounds bearing an isobutyl substituent at the vicinal position of the nitrogen atom of the respective spiro carbocyclic 2,6-DPK residue (1d, $\mathbf{6 d}, \mathbf{7 d}$ ), or compounds possessing a benzylic or 4-chlorobenzylic substituent at the same position of the adamantane-based spiro 2,6-DPK core (1f, $\mathbf{1 g}, \mathbf{2}, \mathbf{3})$, exhibited significant activities against $L$. infantum promastigotes and intracellular amastigotes, in either free base form or hydrochloride salt, with $\mathrm{IC}_{50}$ values at micromolar to low micromolar levels. Their antipromastigote and anti-amastigote activities in the free base form were in the range of 2.67-17.7 $\mu \mathrm{M}$ and 2.23-7.85 $\mu \mathrm{M}$, respectively. Similar ranges of activities against the two forms of this parasite were detected for the corresponding hydrochloride salts (2.86-13.2 $\mu \mathrm{M}$ and 2.04-8.90 $\mu \mathrm{M}$,

This article is protected by copyright. All rights reserved. 
respectively). Moreover, compounds $\mathbf{1 g}$ and $\mathbf{2}$ exhibited micromolar or low micromolar activities against $L$. donovani (Table 2 ). None of the tested compounds showed any significant activity against L. amazonensis promastigotes.

Relative to the parent structures $\mathbf{1 a}, \mathbf{6 a}$ and $\mathbf{7 a}$, the C-isobutyl analogues $\mathbf{1 d}, \mathbf{6 d}$ and $\mathbf{7 d}$, (Senantiomers) were active against the $L$. infantum promastigotes $\left(\mathbf{1 d}, I_{50}=7.23 \mu \mathrm{M}, \mathbf{6 d}, I \mathrm{IC}_{50}=6.16\right.$ $\mu \mathrm{M}$, and $\left.\mathbf{7 d}, \mathrm{IC}_{50}=17.7 \mu \mathrm{M}\right)$. However, they gave better potencies (3.2, 1.8 and 2.3-fold, respectively) toward the intracellular amastigotes, indicating that this form of $L$. infantum is more sensitive to these compounds. In both cases, the cycloheptane-containing C-isobutyl analogue $\mathbf{7 d}$ was less potent than the adamantane and cyclooctane congeners $\mathbf{1 d}$ and $\mathbf{6 d}$, respectively (Table 2 ). No significant activity against the two other Leishmania $s p$. was observed with the isobutyl analogues 1d, $\mathbf{6 d}$ and $\mathbf{7 d}$. As it is evident from Table 2, the adamantane-based C-benzyl or 4-chlorobenzyl substituted analogues $\mathbf{1 f}, \mathbf{1} \mathbf{g}, \mathbf{2}$ and $\mathbf{3}$ were the most efficient growth inhibitors of both forms of $L$. infantum, possessing activities in the low micromolar range (promastigotes form, $\mathrm{IC}_{50}=2.67-4.85 \mu \mathrm{M}$, and amastigote form, $\mathrm{IC}_{50}=2.43-4.40 \mu \mathrm{M}$ ). These analogues in the free base form were 1.5-2.7-fold more effective than the isobutyl counterpart $\mathbf{1} \mathbf{d}$ against promastigotes, while their efficacy toward intracellular amastigotes was comparable to that of $\mathbf{1 d}$. Among the C-benzylated compounds (1f, $\mathbf{1} \mathbf{g}$, 2), the $S$-enantiomer 1 f was the most potent and selective against $L$. infantum exhibiting almost equal anti-promastigote and anti-amastigote potencies. It was 1.4 and 1.7 -fold more active than the corresponding $R$-enantiomer $\mathbf{1 g}$ against promastigotes and intracellular amastigotes, respectively. A similar result against the promastigote form of $L$. infantum was observed when comparing $\mathbf{1 f}$ to the racemic compound 2. Somewhat surprisingly, the $S$-enantiomer 1 f was almost equipotent to the racemic mixture $2\left(\mathbf{1 f}, I_{50}=2.60 \pm 1.37 \mu \mathrm{M} ; \mathbf{2}, I_{50}=2.74 \pm 1.59 \mu \mathrm{M}\right)$ toward intracellular amastigotes, when these compounds were tested as free bases. However, a difference in activity was detected between their respective hydrochlorides, with $\mathbf{1 f}$. $\mathbf{H C l}$ (S-enantiomer) being more potent (2.3-fold) than 2. $\mathbf{H C l}$ (racemic), as expected. Noticeably, enhanced activities were observed against promastigotes of $L$. donovani in the cases of the $R$-enantiomer $\mathbf{1 g}$ and racemate $\mathbf{2}$, when compared to the corresponding $S$-enantiomer $\mathbf{1 f}$. They retained low micromolar anti-promastigote potencies, although the racemic mixture $\mathbf{2}$ was slightly less active. $R$-enantiomer $\mathbf{1 g}$ also exhibited a noteworthy activity toward the intracellular amastigotes in the free base form $\left(I C_{50}=15.0 \mu \mathrm{M}\right)$. These findings indicate that the $R$-stereochemistry is preferred over the $S$ with respect to $L$. donovani potency. Introduction of a chlorine atom at the benzene ring 4-position of the benzylic substituent in the racemic compound $\mathbf{2}$ slightly reduced the potency against $L$. infantum promastigotes, as shown from the $\mathrm{IC}_{50}$ values of compounds $\mathbf{2}$ and $\mathbf{3}$ (Table 2). On the other hand, this chloro-substitution did not significantly affect the activity against the intracellular amastigotes, when the analogues $\mathbf{2}$ and $\mathbf{3}$ were screened as free bases, while a marked difference in potency was detected between the corresponding hydrochloride salts $(2 . \mathrm{HCl}$ and $\mathbf{3 .} \mathbf{H C l})$. In the case of the latter, the hydrochloride salt of the chloro-substituted compound $\mathbf{3}$ was 3.9 times more potent than the hydrochloride salt of its unsubstituted counterpart $\mathbf{2}$.

On the basis of these anti-leishmanial data, the presence of a branched alkyl chain, such as isobutyl group, at the vicinal position of the nitrogen atom of the 2,6-DKP ring of compounds $\mathbf{1 d}, \mathbf{6} \mathbf{d}$ and $\mathbf{7 d}$, or a benzyl substituent at the same position of the adamantane-based compounds $\mathbf{1 f}, \mathbf{1} \mathbf{g}, \mathbf{2}$ and $\mathbf{3}$, is responsible for their enhanced potency. This points to the important influence of these substituents in determining anti-leishmanial activity, possibly due to lipophilic and/or stereoelectronic effects.

This article is protected by copyright. All rights reserved. 
The observed differences in activity (anti-trypanosomal or anti-leishmanial) between the free base and the corresponding hydrochloride salt forms (Table 1 and 2) might be due to their differential cell permeability, resulting from differences in solubility at the $\mathrm{pH}$ of the culture media.

It is noteworthy that the active compounds, show very low cytotoxicity against mammalian cells (rat skeletal myoblast L6 and murine macrophage J 774.1 cell lines). In the case of the myoblast L6 cells, the selectivity indices varied from 63 (1e) to more $>3600$ (1a). With respect to the murine macrophage cells, the $I_{50}$ values were found to be $>200 \mu \mathrm{M}$, with the exception of compound $\mathbf{1 f}$ $\left(\mathrm{IC}_{50}=29.2 \mu \mathrm{M}\right)$. These results indicate significant selectivity of the compounds, up to two orders of magnitude greater than has been reported previously for DKP-based compounds with activity against $T$. brucei ${ }^{[20]}$ If similar properties can be established in vivo, this would represent a significant advantage over current trypanocidal drugs, where toxic side effects are one of the major drawbacks. In this study, despite the lower antileishmanial activity of antimonials- and thus similar activity to the most active hydroxamic acid derivatives, in comparison to amphotericin $\mathrm{B}^{[15,21]}$ (i.e. 135 times lower for Glucantime over amphotericin $\mathrm{B}^{[21]}$ ), we selected not to use antimonials as reference drugs, as parasites may have inherent or even develop spontaneous drug resistance during in vivo or cellculture passages. We thus validated the phenotypic screen by using the highly active amphotericin B. Our results show that the inhibitory activity of Amphotericin $B\left(I_{50}=0.09 \mu \mathrm{M}\right.$ and $0.10 \mu \mathrm{M}$ in $\mathrm{L}$. infantum and $L$. donovani promastigotes respectively and $0.18 \mu \mathrm{M}$ and $0.20 \mu \mathrm{M} L$. infantum and $L$. donovani in intracellular amastigotes respectively) is in the anticipated range. ${ }^{[22]}$

\section{CONCLUSION}

The present work has extended the structure-activity relationships of spiro carbocyclic 2,6-DKP-1acetohydroxamic acids in determining in vitro growth inhibition of trypanosomal and leishmanial parasites. Our studies demonstrate that the anti-parasitic activity of this class of compounds is greatly dependent upon the alkyl or the arylalkyl substitution on either the basic nitrogen atom ( $\mathrm{N}$ methylation) or at its vicinal position ( $C$-alkylation or arylmethylation) in the spiro carbocyclic 2,6DKP skeleton. $\mathrm{N}$-methylation had a positive influence on the potency against blood-stream form $T$. brucei, only in the cases of cyclooctane or cycloheptane-containing analogues (Table 1, compounds 6b, 7b). C-methylation or isobutylation ( $C$-alkylation) reduced potency for the adamantane-based (S)-methyl and (S)-isobutyl substituted analogues $\mathbf{1 c}$ and $\mathbf{1 d}$, respectively. In contrast, enhanced activity against $T$. brucei was observed for the cyclooctane and cycloheptane $(S)$-methyl or $(S)$ isobutyl counterparts $(\mathbf{6 c}, \mathbf{6} \mathbf{d}, \mathbf{7 d})$ ), although the isobutylation produced a more pronounced effect with respect to the methylation. In contrast, $C$-arylmethylation, such as $C$-benzylation or 4chlorobenzylation, yielded $(S)$ or $(R)$-chiral or racemic analogues $(\mathbf{1 f}, \mathbf{1} \mathbf{g}, \mathbf{2}, \mathbf{3})$, which were the most potent against bloodstream-form $T$. brucei, with low nanomolar $\mathrm{IC}_{50}$ values (6.8-32 $\mathrm{nM}$ ) as shown in Table1. Of note, compounds $\mathbf{1 a}, \mathbf{1 b}$ and $\mathbf{1 f}$ displayed significant activity against $T$. cruzi epimastigotes. The $(S)$-chiral benzyl substituted compound $\mathbf{1 f}$ was the most active derivative against T. brucei and T. cruzi, with $\mathrm{IC}_{50}$ values of $6.8 \mathrm{nM}$ and $210 \mathrm{nM}$, respectively. In the case of $T$. cruzi, this value is 5-10 fold lower than the current front line drugs, benznidazole and nifutimox. ${ }^{[23]}$ With respect to the anti-leishmanial activity of the tested compounds, only the $C$-isobutylation or $C$ arylmethylation ( $C$-benzylation or 4-chlorobenzyaltion) produced a significant anti-parasitic toxicity against the $L$. infantum promastigotes and amastigotes (Table 2 , compounds $\mathbf{1 d}, \mathbf{1 f}, \mathbf{1 g}, \mathbf{2}, \mathbf{3}, \mathbf{6 d}$ ). However, this was not the case for the cyclooctane $C$-benzylated analogue 6f. Among the $C$ benzylated compounds $\mathbf{1 f}, \mathbf{1} \mathbf{g}$ and $\mathbf{2}$, the $S$-enantiomer $\mathbf{1} \mathbf{f}$ was the most potent and selective against

This article is protected by copyright. All rights reserved. 
L. infantum. However, the $R$-enantiomer $\mathbf{1 g}$ and the racemate $\mathbf{2}$ displayed satisfactory micromolar activities against $L$. donovani, as compared to the corresponding $S$-enantiomer. These results are indicative of the enhanced potency of the $R$-enantiomer against $L$. donovani. The addition of a chlorine atom at the benzene ring C4-position of the benzylic substituent in the racemic compound $\mathbf{2}$ has a little effect on the potency toward L. infantum (Table 2, 3 vs 2 ).

In summary, the data obtained from this study show that introduction of branched alkyl (e.g. isobutyl) or benzylic substituents at the vicinal position of the amine nitrogen of the 2,6-DKP skeleton tends, in general, to generate analogues with greatly enhanced activity toward trypanosomal and leishmanial parasites. We surmise that this could be due to the strong lipophilic and/or stereoelectronic influence of these bulky substituents in the target binding site. Importantly, the most potent compounds were found not to have significant toxicity against mammalian cells, indicating a high selectivity over the parasites. The results suggest that these acetohydroxamic acid derivatives can be considered promising structures for further investigation in developing new more efficient and safe agents for the treatment of trypanosomiasis or leishmaniasis. Efforts in this direction, including modifications on either the spirocarbocyclic 2,6-DKP residue or the acetohydroxamate acid pharmacophoric moiety, while retaining the carbohydroxamic acid unit (CONHOH), are currently underway.

\section{Figure and Scheme legends}

Figure 1. Structures of lipophilic spiro carbocyclic 2,6-diketopiperazine-1-acetohydroxamic acid derivatives 1a-g, 2-5, 6a-d, 6f, $7 a, 7 b$ and $7 d$.

Scheme 1. Reagents and conditions: (a) $\mathrm{NaCN}$, appropriate $\alpha$-amino acid alkyl ester hydrochloride, $\mathrm{DMSO} / \mathrm{H}_{2} \mathrm{O}$ 29:1 (v/v), rt, $48 \mathrm{~h}$; (b) (i) $\mathrm{H}_{2} \mathrm{SO}_{4} 97 \%$ for 17, 18, 25, rt, 24h or $\mathrm{H}_{2} \mathrm{SO}_{4} 97 \%, \mathrm{CH}_{2} \mathrm{Cl}_{2}, \mathrm{rt}, 24 \mathrm{~h}$ for 19, 20, 26-30 or 48 $\mathrm{h}$ for 21-24; (ii) ice and then aq $\mathrm{NH}_{3} 26 \%$ to $\mathrm{pH} 7-8$; (c) (i) ( $\left.\mathrm{Me}_{3} \mathrm{Si}\right)_{2} \mathrm{NK}$ (1 equiv), THF, 0-5 ${ }^{\circ} \mathrm{C}$, then rt, $1 \mathrm{~h}$, argon; (ii) $\mathrm{BrCH}_{2} \mathrm{CO}_{2} \mathrm{CH}_{2} \mathrm{Ph}$ or $\mathrm{BrCH}_{2} \mathrm{CO}_{2} \mathrm{CH}_{2} \mathrm{C}_{6} \mathrm{H}_{4} \mathrm{OCH}_{3}-4$ only for 38, DMF, rt, $48 \mathrm{~h}$, argon, 78-95\% for 31-37, 38-42, 44, $62 \%$ for 43 from 8; (d) $\mathrm{H}_{2} / \mathrm{Pd}-\mathrm{C} 10 \%$, EtOH for 45, 46, 52, 53, 56, 83-85, 1a-d, 1f, 1g, 2, 4, 5, 6a-d, 6f, 7a, 7b, 7d or EtOH-AcOEt 3:2 (v/v) for 47-51, 54, 55, 57, 1e, 50 psi, rt, $3 \mathrm{~h}$, >99\% for 45-47, 49-57, 83-85, 86\% for 48, 8096\% for 1a-d, 1f, 1g, 2, 4, 5, 6a-d, 6f, 7a, 7b, 7d, 25\% for 1e; (e) (i) CDI, THF for 58-64, 66, 67, 69, 71, 86-88 or THF/DMF 3:4 for 68 or THF/DMF 6:1 for 70, 73 or THF/DMF 4:1 for 72, $28^{\circ} \mathrm{C}, 1 \mathrm{~h}$, argon; (ii) $\mathrm{PhCH}_{2} \mathrm{ONH}_{2} \cdot \mathrm{HCl}$, $\mathrm{Et}_{3} \mathrm{~N}$ or $\mathrm{PhCH}_{2} \mathrm{ONHCH}_{3}$ for $66,67,28^{\circ} \mathrm{C}, 25 \mathrm{~h}$, argon, for $59-63,69-71,73$ or $28^{\circ} \mathrm{C}, 24 \mathrm{~h}$, then $45^{\circ} \mathrm{C}, 1 \mathrm{~h}$, argon for 58, 64, 66-68, 72, 86-88, 58-95\% for 58-60, 62-64, 66-73, 86-88, 62\% for 61 from 34 (f) (i) $\mathrm{CF}_{3} \mathrm{CO}_{2} \mathrm{H}_{1} \mathrm{CH}_{2} \mathrm{Cl}_{2}$, rt, 90 min; (ii) $\mathrm{CDI}, \mathrm{Et}_{3} \mathrm{~N}, \mathrm{THF}, 28^{\circ} \mathrm{C}, 1 \mathrm{~h}$; (iii) $4-\mathrm{CH}_{3} \mathrm{OC}_{6} \mathrm{H}_{4} \mathrm{CH}_{2} \mathrm{ONH}_{2}, 28^{\circ} \mathrm{C}, 24 \mathrm{~h}$, then $55^{\circ} \mathrm{C}, 1 \mathrm{~h}, 70 \%$ from 38; (g) $\mathrm{CF}_{3} \mathrm{CO}_{2} \mathrm{H}, \mathrm{CH}_{2} \mathrm{Cl}_{2}, \mathrm{rt}, 10 \mathrm{~min}$, then $\mathrm{Et}_{3} \mathrm{SiH}, \mathrm{rt}, 45 \mathrm{~min}, 89 \%$ from 65 ; (h) as (c) (i), then $\mathrm{CF}_{3} \mathrm{CO}_{2} \mathrm{H}$ (1 equiv), >99\%; (i) (i) aq $\mathrm{CH}_{2} \mathrm{O} 37 \%, \mathrm{MeOH} / \mathrm{THF}$ 1:1 for 77 or $\mathrm{MeOH} / \mathrm{THF} 1: 3$ for 78, 79, rt, $3 \mathrm{~h}$, then $\mathrm{NaCNBH}_{3}$, rt, $4 \mathrm{~h}$, at pH 6-7 (maintained by adding $\mathrm{AcOH}$ ); (ii) $1 \mathrm{~N} \mathrm{NaOH}$ and $\mathrm{Na}_{2} \mathrm{CO}_{3}$ to $\mathrm{pH} 8,80-92 \%$; (j) $\mathrm{NaH}, \mathrm{DMF}$, rt, $1 \mathrm{~h}$, argon and then as (c) (ii) using $\mathrm{BrCH}_{2} \mathrm{CO}_{2} \mathrm{CH}_{2} \mathrm{Ph}$, $83-92 \%$.

This article is protected by copyright. All rights reserved. 


\section{REFERENCES}

[1] http://www.who.int/mediacentre/factsheets/fs259/en/. Trypanosomiasis, human African (sleeping sickness) (2017).

[2] K. Hashimoto, and K. Yoshioka. Review: surveillance of Chagas disease. Adv. Parasitol. 79 (2012), 375.

[3] http://www.who.int/mediacentre/factsheets/fs375/en/. Leishmaniasis (2016).

[4] S. L. Croft, S. Sundar, A. H. Fairlamb, Clin. Microbiol. Rev. 2006, 19, 111.

[5] A. S. Nagle, S. Khare, A. B. Kumar, F. Supek, A. Buchynskyy, C. J. N. Mathison, N. K. Chennamaneni, N. Pendem, F. S. Buckner, M. H. Gelb, V. Molteni, Chem. Rev. 2014, 114, 11305.

[6] J. Pépin, F. Milord, A. N. Khonde, T. Niyonsenga, L. Loko, B. Mpia, P. De Wals, Trans. R. Soc. Trop. Med. Hyg. 1995, 89, 92.

[7] J. Robays, G. Nyamowala, C. Sese, V. Betu Ku Mesu Kande, P. Lutumba, W. Van der Veken, M. Boelaert, Emerg. Infect. Dis. 2008, 14, 966.

[8] C. Burri, R. Brun, Parasitol. Res. 2003, 90 (Supp 1), S49.

[9] C. Fytas, G. Zoidis, N. Tzoutzas, M.C. Taylor, G. Fytas, J. M. Kelly, J. Med. Chem. 2011, 54, 5250.

[10] A. Tsatsaroni, G. Zoidis, P. Zoumpoulakis, A. Tsotinis, M. C. Taylor, J. M. Kelly, G. Fytas, Tet. Lett. 2013, 54, 3238.

[11] G. Malik, X. Guinchard, D. Crich, Org. Lett. 2012, 14, 596.

[12] C. Fytas, G. Zoidis, G. Fytas, Tetrahedron 2008, 64, 6749.

[13] M. C. Taylor, M. D. Lewis, A. Fortes Francisco, S. R. Wilkinson, J. M. Kelly, PLoS Negl. Trop. Dis. 2015, 9, e0003707.

[14] G. Kendall, A. F. Wilderspin, F. Ashall, M. A. Miles, J. M. Kelly, EMBO J. 1990, 9, 2751.

[15] A. Alexandratos, J. Clos, M. Samiotaki, A. Efstathiou, G. Panayotou, K. Soteriadou, D. Smirlis, Mol. Microbiol. 2013, 88, 1015.

[16] A. Efstathiou, N. Gaboriaud-Kolar, D. Smirlis, V. Myrianthopoulos, K. Vougogiannopoulou, A. Alexandratos, M. Kritsanida, E. Mikros, K. Soteriadou, A.-L. Skaltsounis, Parasit Vector. 2014, $7,1$.

[17] J. F. Oliveiro Costa, A. C. Kiperstok, J. Pereira de Lima David, J. M. David, A. M. Giulietti, L. Paganucci de Queiroz, R. R. dos Santos, M. B. P. Soares, Fitoterapia 2007, 78, 510.

[18] M. Shibata, T. Kashiwamura, A. Yomogita, H. Ishii, M. Sekikawa, H. Shiotani, T. Owada, PCT Int. Appl., 2010067627, 17 Jun 2010.

[19] K. H. Boltze, H. Horstmann, Ger. Offen., 3206885, 15 Sep 1983.

[20] K. R. Watts, J. Ratnam, K. H. Ang, K. Tenney, J. E. Compton, J. McKerrow, P. Crews, Bioorg. Med. Chem. 2010, 18, 2566.

[21] D. C. Ayres, L. A. Pinto, S. Giorgio, J. Parasitol. 2008, 94, 1415.

[22] M. García, R. Scull, P. Satyal, W. N. Setzer, L. Monzote, Phytother. Res. 2017, doi: 10.1002/ptr.5869.

[23] S. R. Wilkinson, C. Bot, J. M. Kelly, B. S. Hall, Curr. Topics Med.Chem. 2011, 16, 2072.

\section{SUPPORTING INFORMATION}

Additional Supporting Information may be found online in the supporting information tab for this article.

This article is protected by copyright. All rights reserved. 


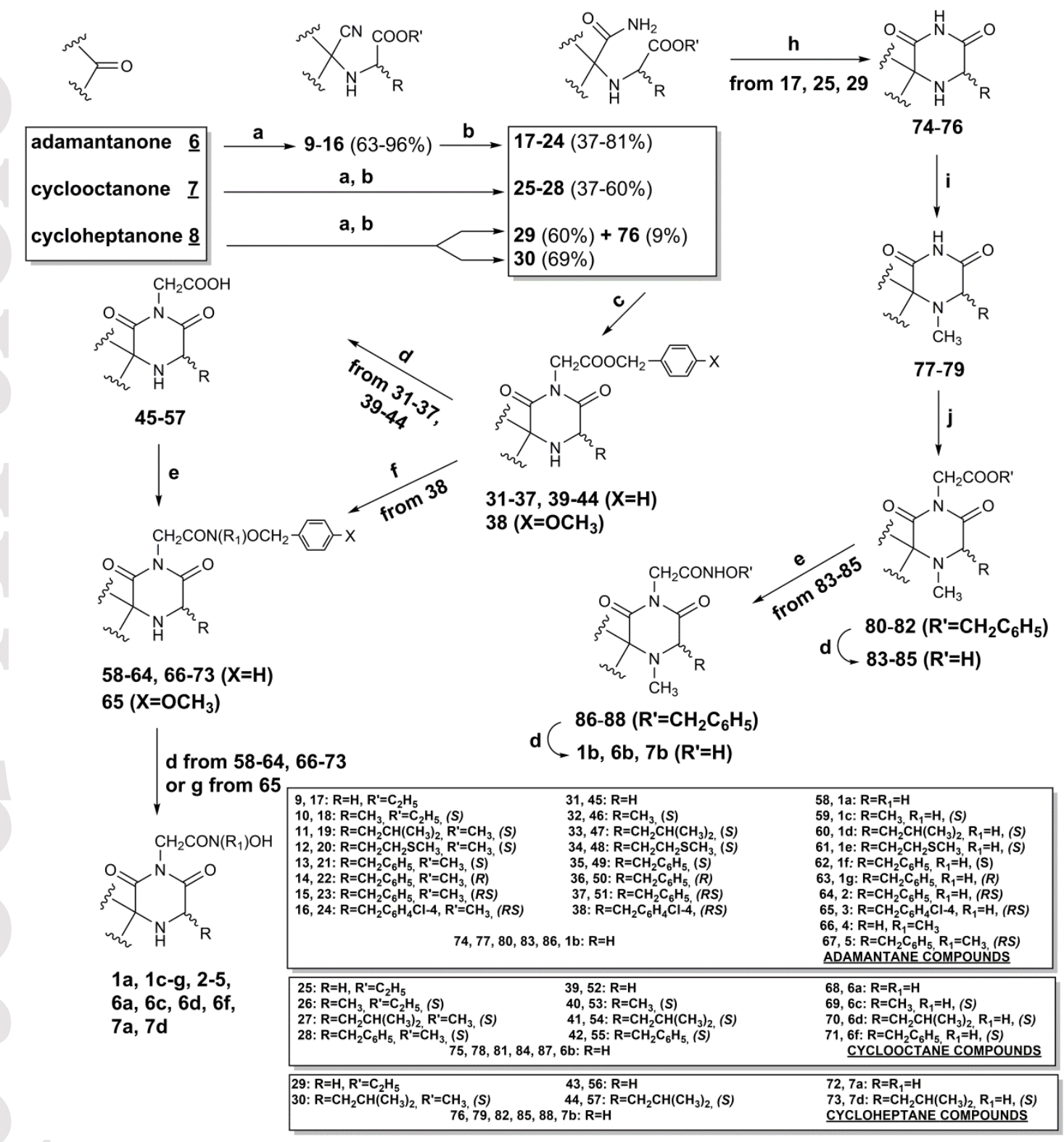

This article is protected by copyright. All rights reserved. 
Table 1. Activity of acetohydroxamic acid analogues 1a-g, 2-5, 6a-d, 6f, 7a, 7b and 7d (Figure 1) tested against cultured bloodstream-form T. brucei $(\mathrm{pH}=7.4)$ and $T$. cruzi epimastigotes, and cytotoxicity of the most active compounds against cultured rat skeletal myoblast L6 cells (Supporting information).

\begin{tabular}{|c|c|c|c|c|c|c|}
\hline \multirow{3}{*}{ Cpds } & \multicolumn{4}{|c|}{ Activity } & \multirow{2}{*}{\multicolumn{2}{|c|}{$\begin{array}{c}\text { Cytotoxicity } \\
\text { L6 cells }\end{array}$}} \\
\hline & \multicolumn{2}{|c|}{ T. brucei } & \multicolumn{2}{|c|}{ T. cruzi } & & \\
\hline & $I C_{50}(n M)^{a, b, c}$ & $I C_{90}(n M)^{a, b, c}$ & $I C_{50}(n M)^{a, b}$ & $I C_{90}(n M)^{a, b}$ & $I C_{50}(n M)^{d}$ & $\mathrm{SI}^{\mathrm{e}}$ \\
\hline $1 a$ & $90 \pm 16(79 \pm 6)^{c}$ & $155 \pm 7(148 \pm 8)^{c}$ & $5510 \pm 680$ & $11110 \pm 1190$ & $>325000$ & $>3600$ \\
\hline 1b & $193 \pm 28(340 \pm 28)$ & $328 \pm 28(622 \pm 84)$ & $3620 \pm 310$ & $5990 \pm 160$ & $40400 \pm 5600$ & 210 \\
\hline 1c & $134 \pm 33(405 \pm 98)$ & $276 \pm 12(909 \pm 209)$ & - & - & - & - \\
\hline 1d & $283 \pm 27(253 \pm 35)$ & $462 \pm 99(355 \pm 17)$ & - & - & $32000 \pm 2000$ & 110 \\
\hline $1 e$ & $168 \pm 42(163 \pm 11)$ & $251 \pm 14(248 \pm 6)$ & - & - & $10500 \pm 500$ & 63 \\
\hline $1 f$ & $6.8 \pm 1.4(42 \pm 5)$ & $11.5 \pm 2(80 \pm 22)$ & $210 \pm 40$ & $360 \pm 10$ & $10400 \pm 900$ & 1500 \\
\hline $1 \mathrm{~g}$ & $9.1 \pm 0.2(9.2 \pm 0.5)$ & $14 \pm 1(14 \pm 1)$ & - & - & $11600 \pm 1500$ & 1300 \\
\hline 2 & $17 \pm 1(18 \pm 1)$ & $26 \pm 3(24 \pm 1)$ & - & - & $23700 \pm 400$ & 1400 \\
\hline 3 & $32 \pm 4(31 \pm 3)$ & $43 \pm 3(40 \pm 2)$ & - & - & $21000 \pm 1000$ & 660 \\
\hline 4 & $246 \times 10^{3}\left(106 \times 10^{3}\right)$ & $523 \times 10^{3}\left(198 \times 10^{3}\right)$ & - & - & - & - \\
\hline 5 & $37 \times 10^{3}\left(35 \times 10^{3}\right)$ & $47 \times 10^{3}\left(45 \times 10^{3}\right)$ & - & - & - & - \\
\hline $6 a$ & $300 \pm 25(266 \pm 19)$ & $635 \pm 14(495 \pm 28)$ & - & - & - & - \\
\hline $6 b$ & $158 \pm 34(162 \pm 15)$ & $300 \pm 34(327 \pm 9)$ & - & - & - & - \\
\hline $6 c$ & $125 \pm 30(134 \pm 13)$ & $270 \pm 41(259 \pm 25)$ & - & - & - & - \\
\hline $6 d$ & $72 \pm 2(73 \pm 3)$ & $92 \pm 9(111 \pm 4)$ & - & - & $204000 \pm 47000$ & 2830 \\
\hline $6 f$ & $29 \pm 3(25 \pm 2)$ & $39 \pm 1(35 \pm 1)$ & - & - & $93800 \pm 18800$ & 3200 \\
\hline $7 a$ & $1870 \pm 80(1150 \pm 130)$ & $\begin{array}{l}2530 \pm 290 \\
(1710 \pm 40)\end{array}$ & - & - & - & - \\
\hline $7 b$ & $285 \pm 9(311 \pm 7)$ & $673 \pm 56(717 \pm 113)$ & - & - & - & - \\
\hline $7 d$ & $99 \pm 4(97 \pm 9)$ & $148 \pm 8(135 \pm 15)$ & - & - & $191000 \pm 2000$ & 1930 \\
\hline
\end{tabular}

Concentrations required to inhibit growth of $T$. brucei and T. cruzi by $50 \%$ and $90 \%$, respectively.

${ }^{b}$ For the active compounds $\mathbf{1 a - g , ~ 2 , ~ 3 , ~ 6 a - d , ~ 6 f , ~ 7 a , ~ 7 b ~ a n d ~} \mathbf{7 d}, I C_{50}$ and $I C_{90}$ data are the mean of triplicate experiments \pm SEM.

${ }^{\mathrm{C}} \mathrm{IC} \mathrm{C}_{50}$ and $\mathrm{IC} \mathrm{C}_{90}$ for the respective hydrochloride are shown in brackets.

This article is protected by copyright. All rights reserved. 
${ }^{d}$ Cytotoxicity was determined by establishing the concentration required to inhibit growth of cultured $\mathrm{L} 6$ cells by $50 \%$ (IC 50 ) (Supporting

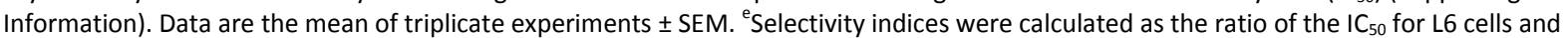
$\mathrm{IC}_{50}$ for T. brucei.

Table 2. In vitro inhibitory activity of acetohydroxamic acid analogues $\mathbf{1 a - g ,} \mathbf{2 , 3}, \mathbf{6 a - d}, \mathbf{6 f}, \mathbf{7 a}, \mathbf{7 b}$ and $\mathbf{7 d}$ (Figure 1) against Leishmania sp. promastigotes and intracellular amastigotes, and their cytotoxicity against murine macrophages $\mathrm{J} 774.1$.

\begin{tabular}{|c|c|c|c|c|c|}
\hline \multirow{2}{*}{ Cpds } & \multicolumn{2}{|c|}{$\begin{array}{l}\text { L. infantum GH12 } \\
\qquad \mathrm{IC}_{50}(\mu \mathrm{M})^{\mathrm{a}, \mathrm{b}, \mathrm{c}}\end{array}$} & \multicolumn{2}{|c|}{$\begin{array}{l}\text { L. donovani Lg13 } \\
\qquad \mathrm{IC}_{50}(\mu \mathrm{M})^{\mathrm{a}, \mathrm{b}, \mathrm{c}}\end{array}$} & \multirow{2}{*}{$\begin{array}{c}\text { Macrophages } \\
\text { J774.1. } \\
\text { IC }_{50}(\mu \mathrm{M})^{d}\end{array}$} \\
\hline & promastigotes & $\begin{array}{l}\text { intracellular } \\
\text { amastigotes }\end{array}$ & promastigotes & $\begin{array}{l}\text { intracellular } \\
\text { amastigotes }\end{array}$ & \\
\hline $1 a$ & ni & - & ni & - & $>200$ \\
\hline $1 b$ & ni & - & $\mathrm{ni}$ & - & $>200$ \\
\hline 1c & ni & - & ni & - & $>200$ \\
\hline $1 d$ & $\begin{array}{c}7.23 \pm 0.25 \\
(7.32 \pm 1.78)^{c}\end{array}$ & $\begin{array}{c}2.23 \pm 1.26 \\
(2.76 \pm 1.11)\end{array}$ & & - & $>200$ \\
\hline $1 e$ & ni & - & ni & - & $>200$ \\
\hline $1 f$ & $\begin{array}{c}2.67 \pm 0.70 \\
(2.86 \pm 0.98)\end{array}$ & $\begin{array}{c}2.60 \pm 1.37 \\
(3.51 \pm 1.20)\end{array}$ & ni & - & $29.3(32)$ \\
\hline $1 g$ & $\begin{array}{c}3.70 \pm 0.23 \\
(2.95 \pm 0.86)\end{array}$ & $\begin{array}{c}4.40 \pm 0.69 \\
(4.70 \pm 0.43)\end{array}$ & $\begin{array}{r}8.35 \pm 0.86 \\
(6.51 \pm 0.32)\end{array}$ & $\begin{array}{c}15 \pm 1.57 \\
(18 \pm 1.34)\end{array}$ & $>200$ \\
\hline 2 & $\begin{array}{r}3.80 \pm 0.46 \\
(4.05 \pm 0.28)\end{array}$ & $\begin{array}{c}2.74 \pm 1.59 \\
(7.90 \pm 0.78)\end{array}$ & $9.73 \pm 0.33(\mathrm{ni})$ & - & $>200$ \\
\hline 3 & $\begin{array}{c}4.85 \pm 1.22 \\
(4.26 \pm 1.39)\end{array}$ & $\begin{array}{c}2.43 \pm 1.18 \\
(2.04 \pm 0.77)\end{array}$ & ni & - & $>200$ \\
\hline $6 a$ & ni & - & ni & - & $>200$ \\
\hline $6 b$ & ni & - & ni & - & $>200$ \\
\hline $6 c$ & ni & - & ni & - & $>200$ \\
\hline $6 d$ & $\begin{array}{c}6.16 \pm 1.29 \\
(5.96 \pm 0.73)\end{array}$ & $\begin{array}{c}3.50 \pm 1.44 \\
(3.20 \pm 1.59)\end{array}$ & ni & - & $>200$ \\
\hline $6 f$ & ni & - & ni & - & $>200$ \\
\hline $7 a$ & ni & - & ni & - & $>200$ \\
\hline $7 b$ & ni & - & ni & - & $>200$ \\
\hline $7 d$ & $\begin{array}{c}17.7 \pm 1.96 \\
(13.2 \pm 0.35)\end{array}$ & $\begin{array}{c}7.85 \pm 1.56 \\
(8.90 \pm 0.46)\end{array}$ & ni & - & $>200$ \\
\hline Amphotericin B & $0.09 \pm 0.005$ & $0.18 \pm 0.02$ & $0.10 \pm 0.01$ & $0.20 \pm 0.02$ & - \\
\hline
\end{tabular}

${ }^{a}$ Concentrations required to inhibit growth of Leishmania sp promastigotes and intracellular amastigotes by $50 \% .{ }^{\mathrm{b}} \mathrm{I}_{50}$ data are the means of triplicate experiments $\pm \mathrm{SEM}$. ${ }^{\mathrm{C}} \mathrm{C}_{50}$ data for the respective hydrochloride are shown in brackets. ${ }^{\mathrm{d} C y t o t o x i c i t y}$ against the murine macrophage cell-line J 774.1. ${ }^{\mathrm{e}} \mathrm{ni}$ - no inhibition at $10 \mu \mathrm{M}$.

This article is protected by copyright. All rights reserved. 


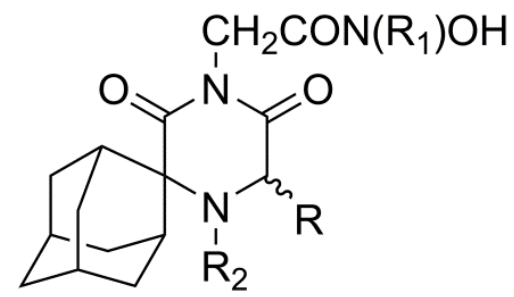

1a-g, 2-5

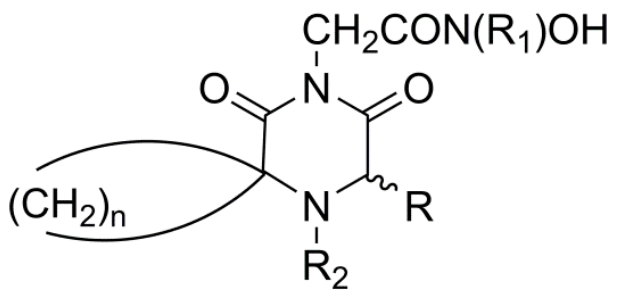

$n=7: 6 a-d, 6 f$

$n=6: 7 a, 7 b, 7 d$
a: $R=R_{1}=R_{2}=H$
b: $\mathrm{R}=\mathrm{R}_{\mathbf{1}}=\mathrm{H}, \mathrm{R}_{\mathbf{2}}=\mathrm{CH}_{\mathbf{3}}$
c: $\mathrm{R}=\mathrm{CH}_{3}, \mathrm{R}_{\mathbf{1}}=\mathrm{R}_{\mathbf{2}}=\mathrm{H}$, (S)-enantiomer
d: $\mathrm{R}=\mathrm{CH}_{2} \mathrm{CH}\left(\mathrm{CH}_{3}\right)_{2}, \mathrm{R}_{1}=\mathrm{R}=\mathrm{H}$, (S)-enantiomer
e: $\mathrm{R}=\mathrm{CH}_{2} \mathrm{CH}_{2} \mathrm{SCH}_{3}, \mathrm{R}_{1}=\mathrm{R}_{2}=\mathrm{H}$, (S)-enantiomer
f: $\mathrm{R}=\mathrm{CH}_{2} \mathrm{C}_{6} \mathrm{H}_{5}, \mathrm{R}_{1}=\mathrm{R}_{2}=\mathrm{H}$, (S)-enantiomer
g: $\mathrm{R}=\mathrm{CH}_{2} \mathrm{C}_{6} \mathrm{H}_{5}, \mathrm{R}_{1}=\mathrm{R}_{2}=\mathrm{H}$, $(R)$-enantiomer
2: $\mathrm{R}=\mathrm{CH}_{2} \mathrm{C}_{6} \mathrm{H}_{5}, \mathrm{R}_{1}=\mathrm{R}_{2}=\mathrm{H}$, racemic
3: $\mathrm{R}=\mathrm{CH}_{2} \mathrm{C}_{6} \mathrm{H}_{4} \mathrm{Cl}-4, \mathrm{R}_{1}=\mathrm{R}_{2}=\mathrm{H}$, racemic
4: $\mathrm{R}=\mathrm{R}_{2}=\mathrm{H}, \mathrm{R}_{1}=\mathrm{CH}_{3}$
5: $\mathrm{R}=\mathrm{CH}_{2} \mathrm{C}_{6} \mathrm{H}_{5}, \mathrm{R}_{1}=\mathrm{CH}_{3}, \mathrm{R}_{2}=\mathrm{H}$, racemic 